\title{
Two-point similarity in the round jet
}

D. EWING ${ }^{1} \dagger$, B. FROHNAPFEL $L^{2}$, W. K. GEORGE , $^{3}$, J. M. PEDERSEN ${ }^{4}$ AND J. WESTER WEEL L

${ }^{1}$ Department of Mechanical Engineering, McMaster University, Hamilton, Ontario, Canada L8S 4M7

${ }^{2}$ Institute of Fluid Mechanics, Friedrich-Alexander University Erlangen-Nuremberg, Erlangen, Germany

${ }^{3}$ Department of Applied Mechanics, Chalmers University of Technology, 41296 Gothenburg, Sweden

${ }^{4}$ Department of Mechnical Engineering, Technical University of Denmark, Lyngby, Denmark

${ }^{5}$ Laboratory for Aero and Hydrodynamics, Delft University of Technology, Leeghwaterstraal 21, 2628 CA Delft, The Netherlands

(Received 30 March 2006 and in revised form 8 November 2006)

The governing equations for the two-point velocity correlations in the far field of the axisymmetric jet are examined and it is shown that these equations can have equilibrium similarity solutions for jets with finite Reynolds number that retain a dependence on the growth rate of the jet. The two-point velocity correlation can be written as the product of a scale that depends on the downstream position of the two points and a function that only depends on the similarity variables. Physically, this result implies that the turbulent processes producing and dissipating energy at the different scales of motion, as well as transferring energy between the different scales of motion, are in equilibrium as the flow evolves downstream. A particularly interesting prediction from the analysis is that the two-point similarity solutions depend only on the separation distance between the points in the streamwise similarity coordinate (i.e. $\left.v=\xi^{\prime}-\xi\right)$, that is, the logarithm of the streamwise coordinate itself (i.e. $\xi=\ln x_{1}$, where $x_{1}$ is measured from a virtual origin). Thus, the measures of the turbulence are homogeneous in the streamwise similarity coordinate.

The predictions from the similarity analysis for the streamwise two-point velocity correlation were compared with combined hot-wire and LDA measurements on the centreline of a round jet at a Reynolds number of 33000 , and with two-point velocity correlations computed from PIV measurements in a round jet at a Reynolds number of 2000 performed by Fukushima et al. In both cases, the measured twopoint velocity correlations in the streamwise direction collapsed when they were scaled in the manner predicted by the similarity analysis. The results provide further evidence that the equilibrium similarity hypothesis does describe the development of the flow in fully developed turbulent round jets and that the two-point correlations are statistically homogeneous in the streamwise similarity coordinate.

\section{Introduction}

There has been considerable interest in characterizing the development of turbulent round jets since these are considered to be among the canonical turbulent free shear

$\dagger$ Current address: Department of Mechanical and Materials Engineering, McLaughlin Hall, Queen's University, Kingston, Ontario, Canada K7L 3N6. 
flows, and are closely related to flows that occur in a broad range of practical applications. Earlier investigations have focused both on the axisymmetric shear layer formed in the near field of the jet, and on the fully developed region of the jet which is of interest here (see Jung, Gamard \& George 2004 and Gamard, Jung \& George 2004 for recent reviews). It is well known that the profiles of the Reynolds-averaged mean velocity field and turbulent stresses become self-similar in the fully developed region of the axisymmetric jet (e.g. Panchapakesan \& Lumley 1993; Hussein, Capp \& George 1994), and this result is often used to estimate the properties of the jet at different downstream locations in practical applications. It has been widely believed until recently that the similarity solutions for the round jet are universal or describe all jets. However, Boersma, Brethouwer \& Nieuwstadt (1998) and Mi, Nobis \& Nathan (2001) found that the Reynolds-averaged profiles of the flow field and scalar field in the fully developed region of the round jet do depend on the initial conditions of the jet, as originally proposed by George $(1989,1995)$ using equilibrium similarity analysis for the single-point equations. A similar dependence on initial conditions has been observed in wakes and shear layer flows (e.g. Wygnanski, Champagne \& Marasli 1986; Ghosal \& Rogers 1997; Slessor, Bond \& Dimotakis 1998; Moser, Rogers \& Ewing 1998; Johansson, George \& Gourlay 2003).

George (1989) argued that the differences observed in the far field could be due to differences in the large-scale structures generated in the near field that propagate into the fully developed region. The large-scale organized motions present in the intermediate and far field of the axisymmetric jet have been characterized in a number of investigations. Many of the earlier investigations (e.g. Dimotakis, Maike-Lye \& Papantoniou 1983; Tso \& Hussain 1989; Mungal \& Hollingsworth 1989) found that motions with aximuthal mode numbers 0 and 1 play a prominent role in the far field, in agreement with the prediction from the stability analysis by Batchelor \& Gill (1962). More recently, Kopiev et al. (1999), Gamard et al. (2002, 2004), and Freund \& Colonius (2002) found that motions with azimuthal mode 2 make the most prominent contribution to the intermediate and far field of the axisymmetric jet and the noise field produced by this flow. Iqbal \& Thomas (2007) found that the azimuthal mode-2 peak occurred when only the streamwise velocity component was considered, but found a mode-1 peak if all three velocity components were included; and this was later confirmed by Wänström, George \& Meyer (2005).

The prominence of the large-scale structures observed in the fully developed region of the axisymmetric jet suggests that the similarity observed in the profiles of the single-point moments is a measure of an underlying 'equilibrium' in the development of the large-scale structures and energy transfer processes in the turbulent flow. Gamard et al. $(2002$, 2004) found that two-point velocity correlations with separation distances in the radial and azimuthal direction on different downstream planes in the fully developed region of the round jet collapsed when they were compared in the similarity variables derived herein, suggesting the structures may be evolving in an equilibrium manner. Gordeyev \& Thomas (2000) had previously found a similar result for correlations with separations in the spanwise and cross-stream direction measured in the plane jet. Neither investigation, however, considered the two-point correlations in the streamwise direction.

Hitherto, there have only been a few attempts to examine if the governing equations for measures of the large-scale structures, such as the two-point velocity correlations, have equilibrium similarity solutions in turbulent flows. Most of these investigations have focused on decaying isotropic turbulence (e.g. von Kármán \& Howarth 1938; Batchelor 1948; George 1992; Speziale \& Bernard 1992) or homogeneous shear 
turbulence (e.g. George \& Gibson 1992). Ewing et al. (2007) did demonstrate that the governing equations for the two-point velocity correlation tensor admit equilibrium similarity solutions in the temporally evolving wake. They also found that the twopoint velocity correlations from two DNS of temporally evolving wakes collapsed when they were scaled in the manner predicted in the analysis, suggesting strongly that any structures present were evolving in an equilibrium manner. With the exception of the recent work of Burattini, Antonia \& Danaila (2005) using structure functions, there do not appear to have been any attempts to extend this analysis to spatially evolving shear flow, such as the axisymmetric jet. In these flows, the characteristic length scales of the structures in the streamwise direction increase as the flow evolves downstream, so the similarity variable for the streamwise direction must be defined differently than for temporally evolving flows.

The present work considers the governing equations for the two-point velocity correlations in the round jet and shows that these equations can have equilibrium similarity solutions. The solutions for the correlations in the streamwise direction are then compared to measurements of the two-point correlation in the streamwise direction that were not considered in the aforementioned investigations. This includes two-point correlations on a jet centreline determined from simultaneous measurements with an LDA system and hot-wire anemometry system. This seems to be the first implementation of this combined technique originally proposed by George, Beuther \& Lumley (1978). Two-point velocity correlations were also computed from the PIV measurements in a jet with a Reynolds number of 2000 performed by Fukushima, Aanen \& Westerweel (2000) that Westerweel et al. (2002) used to examine mixing in the axisymmetric jet. These results do confirm that the predictions from the equilibrium similarity analysis do describe the development of the streamwise two-point velocity correlations in the fully developed region of the axisymmetric jet.

\section{Analysis of the governing equations}

The objective of the analysis is to determine if the governing equations for the two-point velocity correlation tensor can have equilibrium similarity solutions in the axisymmetric jet. These governing equations can be written as (Hinze 1975)

$$
\begin{aligned}
U_{k} \frac{1}{h^{(k)}} & \frac{\partial \overline{u_{i} u_{j}^{\prime}}}{\partial x_{k}}+U_{k}^{\prime} \frac{1}{h^{\prime(k)}} \frac{\partial \overline{u_{i} u_{j}^{\prime}}}{\partial x_{k}^{\prime}}+\frac{U_{2} \overline{u_{3} u_{j}^{\prime}}}{x_{2}} \delta_{i 3}+\frac{U_{2}^{\prime} \overline{u_{i} u_{3}^{\prime}}}{x_{2}^{\prime}} \delta_{j 3} \\
= & -\frac{1}{\rho} \frac{1}{h^{(i)}} \frac{\partial \overline{p u_{j}^{\prime}}}{\partial x_{i}}-\frac{1}{\rho} \frac{1}{h^{\prime(j)}} \frac{\partial \overline{p^{\prime} u_{i}}}{\partial x_{j}^{\prime}}-\frac{1}{h^{(k)}} \frac{\partial \overline{u_{k} u_{i} u_{j}^{\prime}}}{\partial x_{k}}-\frac{1}{h^{\prime}(k)} \frac{\partial \overline{u_{k}^{\prime} u_{i} u_{j}^{\prime}}}{\partial x_{k}^{\prime}} \\
& -\frac{\overline{u_{2} u_{i} u_{j}^{\prime}}}{x_{2}}-\frac{\overline{u_{2}^{\prime} u_{i} u_{j}^{\prime}}}{x_{2}^{\prime}}+\frac{\overline{u_{3}^{2} u_{j}^{\prime}}}{x_{2}} \delta_{i 2}+\frac{\overline{u_{3}^{\prime 2} u_{i}}}{x_{2}^{\prime}} \delta_{j 2}-\frac{\overline{u_{2} u_{3} u_{j}^{\prime}}}{x_{2}} \delta_{i 3}-\frac{\overline{u_{2}^{\prime} u_{i} u_{3}^{\prime}}}{x_{2}^{\prime}} \delta_{j 3} \\
& -\overline{u_{j}^{\prime} u_{k}} \frac{\partial U_{i}}{\partial x_{k}}-\overline{u_{i} u_{k}^{\prime}} \frac{\partial U_{j}^{\prime}}{\partial x_{k}^{\prime}}+v \nabla^{2} \overline{u_{i} u_{j}^{\prime}}+v \nabla^{\prime 2} \overline{u_{i} u_{j}^{\prime}} \\
& -v\left(\frac{\overline{u_{2} u_{j}^{\prime}}}{x_{2}^{2}}+\frac{2}{\frac{\partial}{x_{2}^{2}}} \frac{\overline{u_{3} u_{j}^{\prime}}}{\partial x_{3}}\right) \delta_{i 2}-v\left(\frac{\overline{u_{i} u_{2}^{\prime}}}{x_{2}^{\prime 2}}+\frac{2}{x_{2}^{\prime 2}} \frac{\partial \overline{u_{i} u_{3}^{\prime}}}{\partial x_{3}^{\prime}}\right) \delta_{j 2} \\
& -v\left(\frac{\overline{u_{3} u_{j}^{\prime}}}{x_{2}^{2}}-\frac{2}{x_{2}^{2}} \frac{\partial \overline{u_{2} u_{j}^{\prime}}}{\partial x_{3}}\right) \delta_{i 3}-v\left(\frac{\overline{u_{i} u_{3}^{\prime}}}{x_{2}^{\prime 2}}-\frac{2}{x_{2}^{\prime 2}} \frac{\partial \overline{u_{i} u_{2}^{\prime}}}{\partial x_{3}^{\prime}}\right) \delta_{j 3},
\end{aligned}
$$


where the unprimed variables are evaluated at one location in the jet, and the primed variables are evaluated at a second arbitrary location in the jet at the same point in time. Here, $U_{1}$ and $U_{2}$ are the mean velocities in the streamwise $\left(x_{1}\right)$ and radial $\left(x_{2}\right)$ coordinate directions, while $u_{1}, u_{2}$, and $u_{3}$ are the fluctuating velocities in the streamwise, radial, and azimuthal $\left(x_{3}\right)$ coordinate directions, respectively. Note that the summation convention is not applied to the superscript on the metric for the coordinate system, $h^{(j)}=\left(1,1, x_{2}\right)$. Instead, the superscript has the same value as the index of the differential coordinate next to the metric.

It is proposed that these two-point equations admit equilibrium similarity solutions of the form $\dagger$

$$
\begin{aligned}
\overline{u_{i}\left(x_{1}, x_{2}, x_{3}, t\right) u_{j}^{\prime}\left(x_{1}^{\prime}, x_{2}^{\prime}, x_{3}^{\prime}, t\right)} & =Q_{s}^{(i, j)}\left(x_{1}, x_{1}^{\prime}\right) q_{i, j}\left(v, \eta, \eta^{\prime}, \theta, *\right), \\
\overline{u_{k} u_{i} u_{j}^{\prime}} & =T_{s, 1}^{(k i, j)}\left(x_{1}, x_{1}^{\prime}\right) t_{k i, j}^{1}\left(v, \eta, \eta^{\prime}, \theta, *\right), \\
\overline{u_{k}^{\prime} u_{i} u_{j}^{\prime}} & =T_{s, 2}^{(i, k)}\left(x_{1}, x_{1}^{\prime}\right) t_{i, k j}^{2}\left(v, \eta, \eta^{\prime}, \theta, *\right), \\
\overline{p u_{j}^{\prime}} & =\Pi_{s, 1}^{(, j)}\left(x_{1}, x_{1}^{\prime}\right) \pi_{j}^{1}\left(v, \eta, \eta^{\prime}, \theta, *\right),
\end{aligned}
$$

and

$$
\overline{p^{\prime} u_{i}}=\Pi_{s, 2}^{(i,)}\left(x_{1}, x_{1}^{\prime}\right) \pi_{i}^{2}\left(v, \eta, \eta^{\prime}, \theta, *\right)
$$

where

$$
\begin{aligned}
\eta & =\frac{x_{2}}{\delta\left(x_{1}\right)}, \\
\eta^{\prime} & =\frac{x_{2}^{\prime}}{\delta\left(x_{1}\right)},
\end{aligned}
$$

and

$$
\theta=x_{3}^{\prime}-x_{3}
$$

are the similarity variables in the radial and azimuthal directions, respectively, and $*$ is included to account for any dependence on the initial conditions of the jet. Here, $v$ is a similarity variable for the streamwise direction that will be determined by analysing the governing equations. Thus, the two-point correlations have been written as the product of a scale that depends on the streamwise position of the two points relative to the jet origin, and a function that depends only on the local similarity variables and is independent of the locations of the two points relative to the origin.

The Reynolds-averaged mean continuity and mean momentum equations in the axisymmetric jet contain information about the development of the mean velocity field that is not included in the governing equations for the two-point correlations (or the single-point equations for the Reynolds stresses). Thus, they must also be included in the analysis. Hussein et al. (1994) showed that the mean momentum equations only admit equilibrium similarity solution of the form

$$
U_{1}\left(x_{1}, x_{2}\right)=U_{s}\left(x_{1}\right) f(\eta),
$$

and

$$
\overline{u_{1} u_{2}}=R_{s}\left(x_{1}\right) g(\eta)
$$

$\dagger$ Note that the superscripts on the scales in brackets are not indices, and should not be considered when applying the summation convention. 
when

$$
\begin{aligned}
U_{s}\left(x_{1}\right) & \propto \frac{M_{0}^{1 / 2}}{\delta\left(x_{1}\right)}, \\
\frac{1}{U_{s}\left(x_{1}\right)} \frac{\partial U_{s}}{\partial x_{1}} & \propto \frac{1}{\delta\left(x_{1}\right)} \frac{\mathrm{d} \delta}{\mathrm{d} x_{1}},
\end{aligned}
$$

and

$$
R_{s}\left(x_{1}\right) \propto U_{s}\left(x_{1}\right)^{2} \frac{\mathrm{d} \delta}{\mathrm{d} x_{1}},
$$

while the mean velocity in the radial direction is given by

$$
U_{2}\left(x_{1}, x_{2}\right)=-\left(\left[\delta \frac{\mathrm{d} U_{s}}{\mathrm{~d} x_{1}}\right]+2\left[U_{s} \frac{\mathrm{d} \delta}{\mathrm{d} x_{1}}\right]\right) \frac{1}{\eta} \int_{0}^{\eta} \tilde{\eta} f(\tilde{\eta}) \mathrm{d} \tilde{\eta}+\left[U_{s} \frac{\mathrm{d} \delta}{\mathrm{d} x_{1}}\right] \eta f(\eta) .
$$

Substituting the proposed similarity solutions for the two-point correlations and the solutions for the mean velocities into (2.1) yields equation (A 1) (see Appendix A). The terms in (A 1) have been written as products of functions that depend on the location of the two points relative to the jet origin (in square brackets) and functions that only depend on similarity variables. The equations admit equilibrium similarity solutions of the proposed form if the dependence on the locations of the two points relative to the jet origin can be removed from the equations. This can be accomplished if either all of the terms in square brackets are proportional, or the ratios of different terms only depend on $v$, the similarity variable for the streamwise direction.

The solutions for the pressure-velocity correlations in this incompressible flow must also satisfy

and

$$
\frac{\partial \overline{p u_{1}^{\prime}}}{\partial x_{1}^{\prime}}+\frac{1}{x_{2}^{\prime}} \frac{\partial x_{2}^{\prime} \overline{p u_{2}^{\prime}}}{\partial x_{2}^{\prime}}+\frac{1}{x_{2}^{\prime}} \frac{\partial \overline{p u_{3}^{\prime}}}{\partial x_{3}^{\prime}}=0
$$

$$
\frac{\partial \overline{p^{\prime} u_{1}}}{\partial x_{1}}+\frac{1}{x_{2}} \frac{\partial x_{2} \overline{p^{\prime} u_{2}}}{\partial x_{2}}+\frac{1}{x_{2}} \frac{\partial \overline{p^{\prime} u_{3}}}{\partial x_{3}}=0 .
$$

Substituting the proposed similarity solutions into these equations yields

$$
\begin{aligned}
& {\left[\frac{\partial \Pi_{s, 1}^{(, 1)}}{\partial x_{1}^{\prime}}\right] \pi_{1}^{1}+\left[\Pi_{s, 1}^{(, 1)} \frac{\partial v}{\partial x_{1}^{\prime}}\right] \frac{\partial \pi_{1}^{1}}{\partial v} }-\left[\frac{\Pi_{s, 1}^{(, 1)}}{\delta\left(x_{1}^{\prime}\right)} \frac{\mathrm{d} \delta}{\mathrm{d} x_{1}^{\prime}}\right] \eta^{\prime} \frac{\partial \pi_{1}^{1}}{\partial \eta^{\prime}} \\
&+\left[\frac{\Pi_{s, 1}^{(, 2)}}{\delta\left(x_{1}^{\prime}\right)}\right] \frac{1}{\eta^{\prime}} \frac{\partial \eta^{\prime} \pi_{2}^{1}}{\partial \eta^{\prime}}+\left[\frac{\Pi_{s, 1}^{(, 3)}}{\delta\left(x_{1}^{\prime}\right)}\right] \frac{1}{\eta^{\prime}} \frac{\partial \pi_{3}^{1}}{\partial \theta}=0
\end{aligned}
$$

and

$$
\begin{aligned}
{\left[\frac{\partial \Pi_{s, 2}^{(1,)}}{\partial x_{1}}\right] \pi_{1}^{2}+\left[\Pi_{s, 2}^{(, 1)} \frac{\partial v}{\partial x_{1}}\right] \frac{\partial \pi_{1}^{2}}{\partial v} } & -\left[\frac{\Pi_{s, 2}^{(1,)}}{\delta\left(x_{1}\right)} \frac{\mathrm{d} \delta}{\mathrm{d} x_{1}}\right] \eta \frac{\partial \pi_{1}^{2}}{\partial \eta} \\
+ & {\left[\frac{\Pi_{s, 2}^{(2,)}}{\delta\left(x_{1}\right)}\right] \frac{1}{\eta} \frac{\partial \eta \pi_{2}^{2}}{\partial \eta}-\left[\frac{\Pi_{s, 2}^{(3,)}}{\delta\left(x_{1}\right)}\right] \frac{1}{\eta} \frac{\partial \pi_{3}^{2}}{\partial \theta}=0 . }
\end{aligned}
$$

These equations admit similarity solutions of the proposed form if the terms in square brackets are proportional, or if the ratios of these terms are only a function of $v$.

It can be shown that governing equations for the two-point velocity correlation do indeed admit similarity solutions of the proposed form. The first step in demonstrating 
this is to separate the terms in square brackets in (A 1) into two groups of terms that are proportional; namely,

$$
\begin{aligned}
& {\left[U_{s}\left(x_{1}\right) \frac{\partial Q_{s}^{(i, j)}}{\partial x_{1}}\right] \propto\left[Q_{s}^{(i, j)} U_{s}\left(x_{1}\right) \frac{\partial v}{\partial x_{1}}\right] \propto\left[Q_{s}^{(i, j)} \frac{\mathrm{d} U_{s}\left(x_{1}\right)}{\mathrm{d} x_{1}}\right] \propto\left[\frac{Q_{s}^{(i, j)} U_{s}\left(x_{1}\right)}{\delta\left(x_{1}\right)} \frac{\mathrm{d} \delta}{\mathrm{d} x_{1}}\right]} \\
& \propto\left[\frac{\partial \Pi_{s, 1}^{(, j)}}{\partial x_{1}}\right] \delta_{i 1} \propto\left[\Pi_{s, 1}^{(, j)} \frac{\partial v}{\partial x_{1}}\right] \delta_{i 1} \propto\left[\frac{\Pi_{s, 1}^{(, j)}}{\delta\left(x_{1}\right)} \frac{\mathrm{d} \delta}{\mathrm{d} x_{1}}\right] \delta_{i 1} \propto\left[\frac{\Pi_{s, 1}^{(, j)}}{\delta\left(x_{1}\right)}\right]\left(\delta_{i 2}+\delta_{i 3}\right) \\
& \propto\left[\frac{\partial T_{s, 1}^{(1 i, j)}}{\partial x_{1}}\right] \propto\left[T_{s, 1}^{(1 i, j)} \frac{\partial v}{\partial x_{1}}\right] \propto\left[\frac{T_{s, 1}^{(1 i, j)}}{\delta\left(x_{1}\right)} \frac{\mathrm{d} \delta}{\mathrm{d} x_{1}}\right] \propto\left[\frac{T_{s, 1}^{(2 i, j)}}{\delta\left(x_{1}\right)}\right] \propto\left[\frac{T_{s, 1}^{(3 i, j)}}{\delta\left(x_{1}\right)}\right] \\
& \propto\left[\frac{T_{s, 1}^{(33, j)}}{\delta\left(x_{1}\right)}\right] \delta_{i 2} \propto\left[\frac{T_{s, 1}^{(23, j)}}{\delta\left(x_{1}\right)}\right] \delta_{i 3} \propto\left\{\left[Q_{s}^{(1, j)} \frac{\mathrm{d} U_{s}}{\mathrm{~d} x_{1}}\right] \propto\left[\frac{Q_{s}^{(1, j)} U_{s}\left(x_{1}\right)}{\delta\left(x_{1}\right)} \frac{\mathrm{d} \delta}{\mathrm{d} x_{1}}\right]\right. \\
& \left.\propto\left[\frac{Q_{s}^{(2, j)} U_{s}\left(x_{1}\right)}{\delta\left(x_{1}\right)}\right]\right\} \delta_{i 1} \propto\left\{\left[Q_{s}^{(1, j)} \frac{\mathrm{d} \delta}{\mathrm{d} x_{1}} \frac{\mathrm{d} U_{s}}{\mathrm{~d} x_{1}}\right] \propto\left[Q_{s}^{(1, j)} \delta \frac{\mathrm{d}^{2} U_{s}}{\mathrm{~d} x_{1}^{2}}\right]\right. \\
& \propto\left[Q_{s}^{(1, j)} U_{s}\left(x_{1}\right) \frac{\mathrm{d}^{2} \delta}{\mathrm{d} x_{1}^{2}}\right] \propto\left[Q_{s}^{(1, j)} \frac{\mathrm{d} U_{s}}{\mathrm{~d} x_{1}} \frac{\mathrm{d} \delta}{\mathrm{d} x_{1}}\right] \propto\left[Q_{s}^{(1, j)} \frac{U_{s}\left(x_{1}\right)}{\delta\left(x_{1}\right)}\left(\frac{\mathrm{d} \delta}{\mathrm{d} x_{1}}\right)^{2}\right] \\
& \left.\propto\left[Q_{s}^{(2, j)} \frac{\mathrm{d} U_{s}}{\mathrm{~d} x_{1}}\right] \propto\left[Q_{s}^{(2, j)} \frac{U_{s}\left(x_{1}\right)}{\delta\left(x_{1}\right)} \frac{\mathrm{d} \delta}{\mathrm{d} x_{1}}\right]\right\} \delta_{i 2} \propto\left[\nu \frac{\partial^{2} Q_{s}^{(i, j)}}{\partial x_{1}^{2}}\right] \\
& \propto\left[v \frac{Q_{s}^{(i, j)}}{\delta\left(x_{1}\right)^{2}}\left(\frac{\mathrm{d} \delta}{\mathrm{d} x_{1}}\right)^{2}\right] \propto\left[v \frac{Q_{s}^{(i, j)}}{\delta\left(x_{1}\right)} \frac{\mathrm{d}^{2} \delta}{\mathrm{d} x_{1}^{2}}\right] \propto\left[v \frac{1}{\delta\left(x_{1}\right)} \frac{\mathrm{d} \delta}{\mathrm{d} x_{1}} \frac{\partial Q_{s}^{(i, j)}}{\partial x_{1}}\right] \\
& \propto\left[v \frac{\partial v}{\partial x_{1}} \frac{\partial Q_{s}^{(i, j)}}{\partial x_{1}}\right] \propto\left[v Q_{s}^{(i, j)} \frac{\partial^{2} v}{\partial x_{1}^{2}}\right] \propto\left[v Q_{s}^{(i, j)}\left(\frac{\partial v}{\partial x_{1}}\right)^{2}\right] \\
& \propto\left[v \frac{Q_{s}^{(i, j)}}{\delta} \frac{\mathrm{d} \delta}{\mathrm{d} x_{1}} \frac{\partial v}{\partial x_{1}}\right] \propto\left[v \frac{Q_{s}^{(i, j)}}{\delta\left(x_{1}\right)^{2}}\right]
\end{aligned}
$$

and

$$
\begin{aligned}
{\left[U_{s}\left(x_{1}^{\prime}\right) \frac{\partial Q_{s}^{(i, j)}}{\partial x_{1}^{\prime}}\right] } & \propto\left[Q_{s}^{(i, j)} U_{s}\left(x_{1}^{\prime}\right) \frac{\partial v}{\partial x_{1}^{\prime}}\right] \propto\left[Q_{s}^{(i, j)} \frac{\left.\mathrm{d} U_{s}\left(x_{1}^{\prime}\right)\right]}{\mathrm{d} x_{1}^{\prime}}\right] \propto\left[\frac{Q_{s}^{(i, j)} U_{s}\left(x_{1}^{\prime}\right)}{\delta\left(x_{1}^{\prime}\right)} \frac{\mathrm{d} \delta}{\mathrm{d} x_{1}^{\prime}}\right] \\
& \propto\left[\frac{\partial \Pi_{s, 2}^{(i,)}}{\partial x_{1}^{\prime}}\right] \delta_{j 1} \propto\left[\Pi_{s, 2}^{(i,)} \frac{\partial v}{\partial x_{1}^{\prime}}\right] \delta_{j 2} \propto\left[\frac{\Pi_{s, 2}^{(i,)}}{\delta\left(x_{1}^{\prime}\right)} \frac{\mathrm{d} \delta}{\mathrm{d} x_{1}^{\prime}}\right] \delta_{i 1} \propto\left[\frac{\Pi_{s, 2}^{(i,)}}{\delta\left(x_{1}^{\prime}\right)}\right]\left(\delta_{i 2}+\delta_{i 3}\right) \\
& \propto\left[\frac{\partial T_{s, 2}^{(i, 1 j)}}{\partial x_{1}^{\prime}}\right] \propto\left[T_{s, 2}^{(i, 1 j)} \frac{\partial v}{\partial x_{1}^{\prime}}\right] \propto\left[\frac{T_{s, 2}^{(i, 1)}}{\delta\left(x_{1}^{\prime}\right)} \frac{\mathrm{d} \delta}{\mathrm{d} x_{1}^{\prime}}\right] \propto\left[\frac{T_{s, 2}^{(i, 2 j)}}{\delta\left(x_{1}^{\prime}\right)}\right] \propto\left[\frac{T_{s, 2}^{(i, 3 j)}}{\delta\left(x_{1}^{\prime}\right)}\right] \\
& \propto\left[\frac{T_{s, 2}^{(i, 33)}}{\delta\left(x_{1}^{\prime}\right)}\right] \delta_{j 2} \propto\left[\frac{T_{s, 2}^{(i, 23)}}{\delta\left(x_{1}^{\prime}\right)}\right] \delta_{j 3} \propto\left\{\left[Q_{s}^{(i, 1)} \frac{\mathrm{d} U_{s}}{\mathrm{~d} x_{1}^{\prime}}\right] \propto\left[\frac{Q_{s}^{(i, 1)} U_{s}\left(x_{1}^{\prime}\right)}{\delta\left(x_{1}^{\prime}\right)} \frac{\mathrm{d} \delta}{\mathrm{d} x_{1}^{\prime}}\right]\right. \\
& \left.\propto\left[\frac{Q_{s}^{(i, 2)} U_{s}\left(x_{1}^{\prime}\right)}{\delta\left(x_{1}^{\prime}\right)}\right]\right\} \delta_{i 1} \propto\left\{\left[Q_{s}^{(i, 1)} \frac{\mathrm{d} \delta}{\mathrm{d} x_{1}^{\prime}} \frac{\mathrm{d} U_{s}}{\mathrm{~d} x_{1}^{\prime}}\right] \propto\left[Q_{s}^{(i, 1)} \delta\left(x_{1}^{\prime}\right) \frac{\mathrm{d}^{2} U_{s}}{\mathrm{~d} x_{1}^{\prime 2}}\right]\right. \\
& \propto\left[Q_{s}^{(i, 1)} U_{s}\left(x_{1}^{\prime}\right) \frac{\mathrm{d}^{2} \delta}{\mathrm{d} x_{1}^{\prime 2}}\right] \propto\left[Q_{s}^{(i, 1)} \frac{\mathrm{d} U_{s}}{\mathrm{~d} x_{1}^{\prime}} \frac{\mathrm{d} \delta}{\mathrm{d} x_{1}^{\prime}}\right] \propto\left[Q_{s}^{(i, 1)} \frac{U_{s}\left(x_{1}^{\prime}\right)}{\delta\left(x_{1}^{\prime}\right)}\left(\frac{\mathrm{d} \delta}{\mathrm{d} x_{1}^{\prime}}\right)\right] \\
& \left.\propto\left[Q_{s}^{(i, 2)} \frac{\mathrm{d} U_{s}}{\mathrm{~d} x_{1}^{\prime}}\right] \propto\left[Q_{s}^{(i, 2)} \frac{U_{s}\left(x_{1}^{\prime}\right)}{\delta\left(x_{1}^{\prime}\right)} \frac{\mathrm{d} \delta}{\mathrm{d} x_{1}^{\prime}}\right]\right\} \delta_{j 2} \propto\left[v \frac{\partial^{2} Q_{s}^{(i, j)}}{\partial x_{1}^{\prime 2}}\right]
\end{aligned}
$$




$$
\begin{aligned}
& \propto\left[v \frac{Q_{s}^{(i, j)}}{\delta\left(x_{1}\right)^{\prime 2}}\left(\frac{\mathrm{d} \delta}{\mathrm{d} x_{1}^{\prime}}\right)^{2}\right] \propto\left[v \frac{Q_{s}^{(i, j)}}{\delta\left(x_{1}^{\prime}\right)} \frac{\mathrm{d}^{2} \delta}{\mathrm{d} x_{1}^{\prime 2}}\right] \propto\left[v \frac{1}{\delta\left(x_{1}^{\prime}\right)} \frac{\mathrm{d} \delta}{\mathrm{d} x_{1}^{\prime}} \frac{\partial Q_{s}^{(i, j)}}{\partial x_{1}^{\prime}}\right] \\
& \propto\left[v \frac{\partial v}{\partial x_{1}^{\prime}} \frac{\partial Q_{s}^{(i, j)}}{\partial x_{1}^{\prime}}\right] \propto\left[\nu Q_{s}^{(i, j)} \frac{\partial^{2} v}{\partial x_{1}^{\prime 2}}\right] \propto\left[\nu Q_{s}^{(i, j)}\left(\frac{\partial v}{\partial x_{1}^{\prime}}\right)^{2}\right] \\
& \propto\left[v \frac{Q_{s}^{(i, j)}}{\delta} \frac{\mathrm{d} \delta}{\mathrm{d} x_{1}} \frac{\partial v}{\partial x_{1}^{\prime}}\right] \propto\left[v \frac{Q_{s}^{(i, j)}}{\delta\left(x_{1}^{\prime}\right)^{2}}\right] .
\end{aligned}
$$

The constraints in (2.20) and (2.21) are satisfied when the scales for the similarity solutions are given by

$$
\begin{aligned}
Q_{s}^{(i, j)}\left(x_{1}, x_{1}^{\prime}\right) & =a_{c}^{(i, j)}(*) U_{s}\left(x_{1}\right) U_{s}\left(x_{1}^{\prime}\right), \\
T_{s, 1}^{(k i, j)}\left(x_{1}, x_{1}^{\prime}\right) & =b_{c, 1}^{(k i, j)}(*) U_{s}\left(x_{1}\right) Q_{s}^{(i, j)}\left(x_{1}, x_{1}^{\prime}\right), \\
T_{s, 2}^{(i, k j)}\left(x_{1}, x_{1}^{\prime}\right) & =b_{c, 2}^{(i, k j)}(*) U_{s}\left(x_{1}^{\prime}\right) Q_{s}^{(i, j)}\left(x_{1}, x_{1}^{\prime}\right), \\
\Pi_{s, 1}^{(, j)}\left(x_{1}, x_{1}^{\prime}\right) & =d_{c, 1}^{(, j)}(*) U_{s}\left(x_{1}\right) Q_{s}^{(i, j)}\left(x_{1}, x_{1}^{\prime}\right),
\end{aligned}
$$

and

$$
\Pi_{s, 2}^{(i,)}\left(x_{1}, x_{1}^{\prime}\right)=d_{c, 2}^{(i,)}(*) U_{s}\left(x_{1}^{\prime}\right) Q_{s}^{(i, j)}\left(x_{1}, x_{1}^{\prime}\right),
$$

where $a_{c}^{(i, j)}(*), b_{c, 1}^{(k i, j)}(*), b_{c, 2}^{(i, k j)}(*), d_{c, 1}^{(, j)}(*)$, and $d_{c, 2}^{(i,)}(*)$ are parameters that can depend on the source conditions of the flow. The constraints in (2.20) and (2.21) and the equations for the pressure-velocity correlation are only satisfied when

$$
\frac{\mathrm{d} \delta}{\mathrm{d} x_{1}}=\text { constant }
$$

and the streamwise similarity variable satisfies

$$
\frac{\partial v}{\partial x_{1}} \propto \frac{1}{\delta\left(x_{1}\right)} \frac{\mathrm{d} \delta}{\mathrm{d} x_{1}} \quad \text { and } \quad \frac{\partial v}{\partial x_{1}^{\prime}} \propto \frac{1}{\delta\left(x_{1}^{\prime}\right)} \frac{\mathrm{d} \delta}{\mathrm{d} x_{1}^{\prime}} .
$$

Therefore, it follows that

$$
\frac{\partial v}{\partial x_{1}} \propto \frac{1}{x_{1}} \text { and } \quad \frac{\partial v}{\partial x_{1}^{\prime}} \propto \frac{1}{x_{1}^{\prime}} .
$$

or that

$$
v=\alpha_{1} \ln x_{1}+\beta_{1}\left(x_{1}^{\prime}\right) \text { and } v=\alpha_{2} \ln x_{1}+\beta_{2}\left(x_{1}\right),
$$

where $\alpha_{1}$ and $\alpha_{2}$ are constants, and $\beta_{1}$ and $\beta_{2}$ are functions that depend on $x_{1}^{\prime}$ and $x_{1}$, respectively. These results can be combined to yield

$$
v=\alpha_{1} \ln x_{1}+\alpha_{2} \ln x_{1}^{\prime}+\gamma_{1},
$$

where $\gamma_{1}$ is a constant.

The constants in (2.31) can be determined by examining the ratios of the terms in (2.20) to those in (2.21) that are proportional to

$$
\left[\frac{Q_{s}^{(i, j)} U_{s}\left(x_{1}\right)}{\delta\left(x_{1}\right)} \frac{\mathrm{d} \delta}{\mathrm{d} x_{1}}\right] /\left[\frac{Q_{s}^{(i, j)} U_{s}\left(x_{1}^{\prime}\right)}{\delta\left(x_{1}^{\prime}\right)} \frac{\mathrm{d} \delta}{\mathrm{d} x_{1}}\right] \propto \frac{x_{1}^{\prime 2}}{x_{1}^{2}} .
$$

The equations for the two-point velocity correlations only admit similarity solutions of the proposed form if these ratios are constants or functions of $v$; i.e.

$$
\frac{x_{1}^{\prime 2}}{x_{1}^{2}}=\Gamma(v)
$$


or

$$
\ln \left(x_{1}^{\prime}\right)-\ln \left(x_{1}\right)=\frac{\ln \Gamma(v)}{2}=\Phi(v) .
$$

Thus, it follows that $\alpha_{1}=-\alpha_{2}$ and $\gamma_{1}=0 . \dagger$ Here, $\alpha_{2}$ is set equal to 1 , so that

$$
v=\ln x_{1}^{\prime}-\ln x_{1} .
$$

This can be written as

$$
v=\xi^{\prime}-\xi
$$

where

$$
\xi=\ln x_{1}
$$

is the similarity coordinate in the streamwise direction. $\dagger$ This transformation changes the semi-infinite streamwise coordinate $x_{1}$ into a coordinate $\xi$ that is infinite in extent.

Thus, the governing equations for the two-point velocity correlations do indeed admit equilibrium similarity solutions of the proposed form. Substituting the similarity solutions into the equations for the two-point velocity correlations yields

$$
\begin{aligned}
& \mathrm{e}^{v} a_{c}^{(i, j)} {\left[-f(\eta) q_{i, j}-f(\eta) \frac{\partial q_{i, j}}{\partial v}-\left(\frac{\partial q_{i, j}}{\partial \eta}+\frac{q_{3, j}}{\eta} \delta_{i 3}\right) \frac{1}{\eta} \int_{0}^{\eta} \tilde{\eta} f(\tilde{\eta}) \mathrm{d} \tilde{\eta}+f(\eta) q_{3, j} \delta_{i 3}\right] } \\
& \mathrm{e}^{-v} a_{c}^{(i, j)}\left[-f\left(\eta^{\prime}\right) q_{i, j}+f\left(\eta^{\prime}\right) \frac{\partial q_{i, j}}{\partial v}-\left(\frac{\partial q_{i, j}}{\partial \eta^{\prime}}+\frac{q_{i, 3}}{\eta^{\prime}} \delta_{j 3}\right) \frac{1}{\eta^{\prime}} \int_{0}^{\eta^{\prime}} \tilde{\eta} f(\tilde{\eta}) \mathrm{d} \tilde{\eta}+f\left(\eta^{\prime}\right) q_{i, 3} \delta_{j 3}\right] \\
&\left.\left.=+\frac{\mathrm{e}^{v}}{\rho} d_{c, 1}^{(, j)}\left\{\left[2 \pi_{j}^{1}+\eta \frac{\partial p_{j}^{1}}{\partial \eta}+\frac{\partial p_{j}^{1}}{\partial v}\right] \delta_{i 1}-\left(\frac{\mathrm{d} \delta}{\mathrm{d} x_{1}}\right)^{-1}\right] \frac{\partial \pi_{j}^{1}}{\partial \eta} \delta_{i 2}-\frac{1}{\eta} \frac{\partial \pi_{j}^{1}}{\partial \theta} \delta_{i 3}\right]\right\} \\
&+\frac{\mathrm{e}^{-v}}{\rho} d_{c, 2}^{(i,)}\left\{\left[2 \pi_{i}^{2}+\eta \frac{\partial p_{i}^{2}}{\partial \eta^{\prime}}-\frac{\partial p_{j}^{1}}{\partial v}\right] \delta_{j 1}-\left(\frac{\mathrm{d} \delta}{\mathrm{d} x_{1}}\right)^{-1}\left[\frac{\partial \pi_{i}^{2}}{\partial \eta^{\prime}} \delta_{j 2}+\frac{1}{\eta^{\prime}} \frac{\partial \pi_{i}^{2}}{\partial \theta} \delta_{j 3}\right]\right\} \\
&+\mathrm{e}^{v}\left[b_{c, 1}^{(1 i, j)}\left(2 t_{1 i, j}^{1}+\eta \frac{\partial t_{1 i, j}^{1}}{\partial \eta}+\frac{\partial t_{1 i, j}^{1}}{\partial v}\right)-b_{c, 1}^{(2 i, j)} \frac{1}{\eta} \frac{\partial \eta t_{2 i, j}^{1}}{\partial \eta}+b_{c, 1}^{(3 i, j)} \frac{1}{\eta} \frac{\partial t_{3 i, j}^{1}}{\partial \theta}\right. \\
&\left.+b_{c, 1}^{(33, j)}(*) \frac{t_{33, j}^{1}}{\eta} \delta_{i 2}-b_{c, 1}^{(23, j)} \frac{t_{23, j}^{1}}{\eta} \delta_{i 3}\right]+\mathrm{e}^{-v}\left[b_{c, 2}^{(i, 1 j)}\left(2 t_{i, 1 j}^{2}+\eta^{\prime} \frac{\partial t_{i, 1 j}^{2}}{\partial \eta^{\prime}}-\frac{\partial t_{i, 1 j}^{2}}{\partial v}\right)\right. \\
&\left.\quad-b_{c, 2}^{(i, 2, j)} \frac{1}{\eta^{\prime}} \frac{\partial \eta^{\prime} t_{i, 2 j}^{2}}{\partial \eta^{\prime}}-b_{c, 2}^{(i, 3 j)} \frac{1}{\eta^{\prime}} \frac{\partial t_{i, 3 j}^{2}}{\partial \theta}+b_{c, 2}^{(i, 33)} \frac{t_{i, 33}^{2}}{\eta^{\prime}} \delta_{j 2}-b_{c, 2}^{(i, 23)} \frac{t_{i, 23}^{1}}{\eta^{\prime}} \delta_{j 3}\right] \\
&+\mathrm{e}^{v}\left[\left\{a_{c}^{(1, j)} q_{1, j}\left(f(\eta)+\eta \frac{\mathrm{d} f}{\mathrm{~d} \eta}\right)-\frac{a_{c}^{(2, j)}}{\mathrm{d} \delta / \mathrm{d} x_{1}} q_{2, j} \frac{\mathrm{d} f}{\mathrm{~d} \eta}\right\} \delta_{i 1}\right. \\
&\left.+\left\{a_{c}^{(1, j)} \frac{\mathrm{d} \delta}{\mathrm{d} x_{1}} q_{1, j} \eta \frac{\mathrm{d} \eta f}{\mathrm{~d} \eta}-a_{c}^{(2, j)} q_{2, j}\left(\eta \frac{\mathrm{d} f}{\mathrm{~d} \eta}+\frac{1}{\eta^{2}} \int_{0}^{\eta} \tilde{\eta} f d \tilde{\eta}\right)\right\} \delta_{i 2}\right]
\end{aligned}
$$

$\dagger$ Note that as a result, $U_{s}\left(x_{1}\right)=\mathrm{e}^{v} U_{s}\left(x_{1}^{\prime}\right)$, so there is a variety of ways to represent the dependence on the location of the two points in (2.22)-(2.26).

$\dagger$ The similarity coordinate in the streamwise direction could have been deduced intuitively by recognizing that the similarity coordinates re-scale the physical coordinates to account for changes in the size of the turbulent motions as the flow evolves downstream. Thus, $\xi$ can be defined as $\mathrm{d} \xi \propto \mathrm{d} x_{1} / \delta_{1}\left(x_{1}\right)$. If $\delta_{1}\left(x_{1}\right) \propto \delta\left(x_{1}\right)$ and $\delta\left(x_{1}\right) \propto x_{1}$, it follows that $\xi=\ln \left(x_{1}\right)$. The proposed similarity solutions must be independent of the two points' locations relative to the jet origin, and thus could only be a function of the separation distance between the two points in the similarity coordinate; i.e. $v=\xi^{\prime}-\xi$, as deduced from the analysis of the equations above. 


$$
\begin{aligned}
& +\mathrm{e}^{-v}\left[\left\{a_{c}^{(i, 1)} q_{i, 1}\left(f\left(\eta^{\prime}\right)+\eta^{\prime} \frac{\mathrm{d} f}{\mathrm{~d} \eta^{\prime}}\right)-\frac{a_{c}^{(i, 2)}}{\mathrm{d} \delta / \mathrm{d} x_{1}^{\prime}} q_{i, 2} \frac{\mathrm{d} f}{\mathrm{~d} \eta^{\prime}}\right\} \delta_{j 1}\right. \\
& \left.+\left\{a_{c}^{(i, 1)}\left(\frac{\mathrm{d} \delta}{\mathrm{d} x_{1}^{\prime}}\right) q_{i, 1} \eta^{\prime} \frac{\mathrm{d} \eta^{\prime} f}{\mathrm{~d} \eta^{\prime}}-a_{c}^{(i, 2)} q_{i, 2}\left(\eta^{\prime} \frac{\mathrm{d} f}{\mathrm{~d} \eta^{\prime}}+\frac{1}{\eta^{\prime 2}} \int_{0}^{\eta^{\prime}} \tilde{\eta} f \mathrm{~d} \tilde{\eta}\right)\right\} \delta_{j 2}\right] \\
& +\mathrm{e}^{v}\left[\frac{a_{c}^{(i, j)}}{R e_{\delta}} \frac{\mathrm{d} \delta}{\mathrm{d} x_{1}}\right]\left[2 q_{i, j}+4 \eta \frac{\partial q_{i, j}}{\partial \eta}+3 \frac{\partial q_{i, j}}{\partial v}+2 \eta \frac{\partial^{2} q_{i, j}}{\partial \eta \partial v}+\eta^{2} \frac{\partial^{2} q_{i, j}}{\partial \eta^{2}}+\frac{\partial^{2} q_{i, j}}{\partial v^{2}}\right] \\
& +\mathrm{e}^{v}\left[\frac{1}{R e_{\delta}}\left(\frac{\mathrm{d} \delta}{\mathrm{d} x_{1}}\right)^{-1}\right]\left\{a_{c}^{(i, j)}\left(\frac{1}{\eta} \frac{\partial}{\partial \eta} \eta \frac{\partial q_{i, j}}{\partial \eta}+\frac{1}{\eta^{2}} \frac{\partial^{2} q_{i, j}}{\partial \theta^{2}}\right)\right. \\
& \left.-\left(a_{c}^{(2, j)} \frac{q_{2, j}}{\eta^{2}}-\frac{2 a_{c}^{(3, j)}}{\eta^{2}} \frac{\partial q_{3, j}}{\partial \theta}\right) \delta_{i 2}-\left(a_{c}^{(3, j)} \frac{q_{3, j}}{\eta^{2}}+\frac{2 a_{c}^{(2, j)}}{\eta^{2}} \frac{\partial q_{2, j}}{\partial \theta}\right) \delta_{i 3}\right\} \\
& +\mathrm{e}^{-v}\left[\frac{a_{c}^{(i, j)}}{R e_{\delta}} \frac{\mathrm{d} \delta}{\mathrm{d} x_{1}}\right]\left\{2 q_{i, j}+4 \eta^{\prime} \frac{\partial q_{i, j}}{\partial \eta^{\prime}}-3 \frac{\partial q_{i, j}}{\partial v}-2 \eta^{\prime} \frac{\partial^{2} q_{i, j}}{\partial \eta^{\prime} \partial v}+\eta^{\prime 2} \frac{\partial^{2} q_{i, j}}{\partial \eta^{\prime 2}}+\frac{\partial^{2} q_{i, j}}{\partial v^{2}}\right\} \\
& +\mathrm{e}^{-v}\left[\frac{1}{R e_{\delta}}\left(\frac{\mathrm{d} \delta}{\mathrm{d} x_{1}}\right)^{-1}\right]\left\{a_{c}^{(i, j)}\left(\frac{1}{\eta^{\prime}} \frac{\partial}{\partial \eta^{\prime}} \eta^{\prime} \frac{\partial q_{i, j}}{\partial \eta^{\prime}}+\frac{1}{\eta^{\prime 2}} \frac{\partial^{2} q_{i, j}}{\partial \theta^{2}}\right)\right. \\
& \left.-\left(a_{c}^{(i, 2)} \frac{q_{i, 2}}{\eta^{\prime 2}}+\frac{2 a_{c}^{(i, 3)}}{\eta^{\prime 2}} \frac{\partial q_{i, 3}}{\partial \theta}\right) \delta_{i 2}-\left(a_{c}^{(i, 3)} \frac{q_{i, 3}}{\eta^{\prime 2}}-\frac{2 a_{c}^{(i, 2)}}{\eta^{\prime 2}} \frac{\partial q_{i, 2}}{\partial \theta}\right) \delta_{j 3}\right\},
\end{aligned}
$$

while the equations for the pressure velocity correlation are given by

$$
-2 d_{c, 1}^{(, 1)} \pi_{1}^{1}+d_{c, 1}^{(, 1)} \frac{\partial \pi_{1}^{1}}{\partial v}-d_{c, 1}^{(, 1)} \eta^{\prime} \frac{\partial \pi_{1}^{1}}{\partial \eta^{\prime}}+\frac{d_{c, 1}^{(, 2)}}{\mathrm{d} \delta / \mathrm{d} x_{1}^{\prime}} \frac{1}{\eta^{\prime}} \frac{\partial \eta^{\prime} \pi_{2}^{1}}{\partial \eta^{\prime}}+\frac{d_{c, 1}^{(, 3)}}{\mathrm{d} \delta / \mathrm{d} x_{1}^{\prime}} \frac{1}{\eta^{\prime}} \frac{\partial \pi_{3}^{1}}{\partial \theta}=0
$$

and

$$
-2 d_{c, 2}^{(1,)} \pi_{1}^{2}-d_{c, 2}^{(1,)} \frac{\partial \pi_{1}^{2}}{\partial v}-d_{c, 2}^{(1,)} \eta \frac{\partial \pi_{1}^{2}}{\partial \eta}+\frac{d_{c, 2}^{(2,)}}{\mathrm{d} \delta / \mathrm{d} x} \frac{1}{\eta} \frac{\partial \eta \pi_{2}^{2}}{\partial \eta}-\frac{d_{c, 2}^{(3,)}}{\mathrm{d} \delta / \mathrm{d} x} \frac{1}{\eta} \frac{\partial \pi_{3}^{2}}{\partial \theta}=0,
$$

where the Reynolds number

$$
R e_{\delta}=\frac{U_{s}\left(x_{1}\right) x_{1}}{v} \propto \frac{U_{s} \delta}{v}
$$

and the growth rate $\mathrm{d} \delta / \mathrm{d} x_{1}$ are both constants that may depend on the source conditions of the jet. There is no choice of $a_{c}^{(i, j)}$ that will remove the Reynolds number from both the convective and viscous terms in the equations. Thus, the equations admit similarity solutions even for finite-Reynolds-number jets, but the solution depends on the jet Reynolds number. There is also no choice for $a_{c}^{(i, j)}$ that will remove the growth-rate-dependent parameter from these equations. In particular, comparing the convection, pressure-velocity transfer, and the dominant production terms in the $\overline{u_{1} u_{1}^{\prime}}$ equation, it is clear that this equation can only be independent of $\mathrm{d} \delta / \mathrm{d} x$ if

$$
\frac{d_{c, 1}^{(, 1)}}{a_{c}^{(1,1)}}=\mathrm{const}
$$

and

$$
\frac{a_{c}^{(2,1)}(\mathrm{d} \delta / \mathrm{d} x)^{-1}}{a_{c}^{(1,1)}}=\mathrm{const}
$$

where the constants must be independent of the jet growth rate. Similarly, the $\overline{u_{2} u_{2}^{\prime}}$ equation, the $\overline{u_{2} u_{1}^{\prime}}$ equation, and the pressure-velocity correlation equation could only 
be independent of $\mathrm{d} \delta / \mathrm{d} x_{1}$ if

$$
\begin{aligned}
\frac{d_{c, 1}^{(, 2)}\left(\mathrm{d} \delta / \mathrm{d} x_{1}\right)^{-1}}{a_{c}^{(2,2)}} & =\text { const }, \\
\frac{a_{c}^{(2,2)}\left(\mathrm{d} \delta / \mathrm{d} x_{1}\right)^{-1}}{a_{c}^{(2,1)}} & =\text { const },
\end{aligned}
$$

and

$$
\frac{d_{c, 1}^{(, 2)}\left(\mathrm{d} \delta / \mathrm{d} x_{1}^{\prime}\right)^{-1}}{d_{c, 1}^{(, 1)}}=\text { const. }
$$

Equations (2.42), (2.44), and (2.46) can only be satisfied if $a_{c}^{(1,1)} / a_{c}^{(2,2)}$ is independent of the jet growth rate, while (2.43) and (2.45) can only be satisfied if $a_{c}^{(1,1)}\left(\mathrm{d} \delta / \mathrm{d} x_{1}\right)^{2} / a_{c}^{(2,2)}$ is independent of the jet growth rate. These cannot both be satisfied, so there is no choice for the parameters that would remove the dependence on the jet growth rate from the governing equations for the two-point similarity solutions. Thus there is no unique similarity solution for the two-point velocity correlations in the axisymmetric jet, an apparently universal characteristic of free turbulent flows.

It can only be determined from experiment whether or not the two-point similarity solutions deduced here describe the evolution of actual jets. Gamard et al. (2002, 2004) showed that two-point velocity correlations measured on different downstream planes in the fully developed region of the axisymmetric jet with separations in only the azimuthal and radial directions change in a manner consistent with the predictions of the equilibrium similarity analysis. Gordeyev \& Thomas (2000) also found a similar result for two-point correlations in the plane jet, again for the case when the separations were only in lateral and cross-stream directions. Hitherto, however, there do not appear to have been any measurements of the two-point correlation with separations in the streamwise direction at different downstream locations in the fully developed region of the round jet that could be used to determine if the two-point similarity solutions deduced here do describe the change in the streamwise correlations in actual jets. Of particular interest, of course, is the prediction that the two-point correlation is statistically homogeneous in the streamwise similarity coordinate, $\xi$.

\section{Experimental methodology and uncertainty}

Experiments were performed here (described in detail below) to measure the twopoint velocity correlation of the streamwise velocity along the centreline of a jet with an exit Reynolds number of 33000 , using an LDA system to measure the upstream velocity and a hot-wire to measure the velocity at the downstream locations. The two-point correlations in the streamwise direction in an axisymmetric jet at a Reynolds number of 2000 were also computed from PIV measurements performed by Fukushima et al. (2000) and discussed in Westerweel et al. (2002). This database was used here to compute the streamwise two-point correlations of two velocity components (streamwise and radial) both on and off the jet centreline. The experimental methodology for the experiment performed here is described in detail first. The uncertainties in the two-point correlations determined using the LDA/hot-wire technique and from the database of PIV measurements are then discussed in detail. 


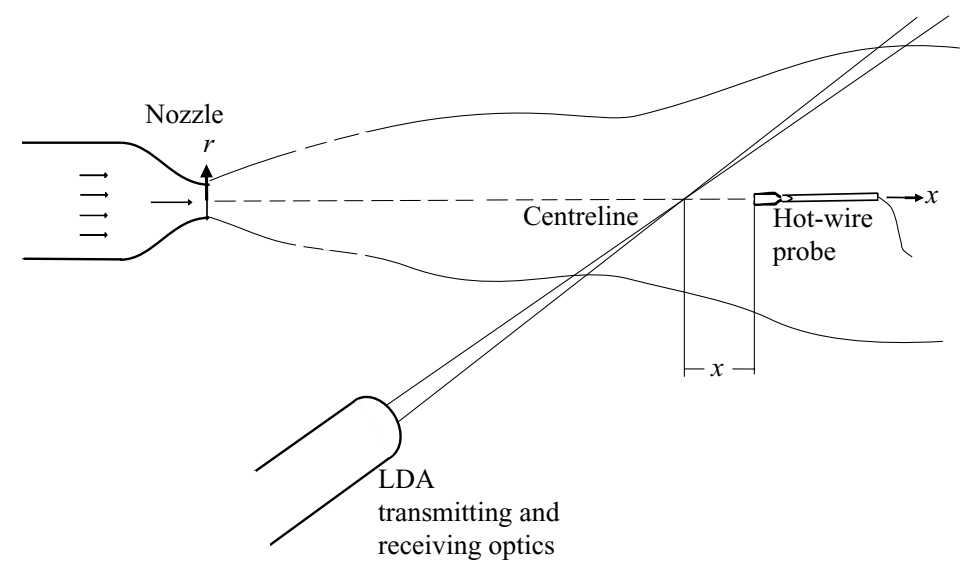

FIGURE 1. Schematic showing the set-up of the hot-wire and LDA during the measurements.

\subsection{Chalmers experiment}

The experimental facility used in this investigation was used previously by Gamard et al. (2004), and designed as a $2 / 5$-scale replica of the facility used by Hussein et al. (1994). The flow from a blower was conditioned in a $58.5 \mathrm{~cm} \times 58.5 \mathrm{~cm} \times 59 \mathrm{~cm}$ settling chamber before entering a contoured nozzle with an exit diameter of $1 \mathrm{~cm}$. The jet exited into an enclosure that was large enough to minimize the return flow. The momentum of the forward jet flow was conserved to within a few per cent at 150 diameters downstream of the jet exit. The development of the jet in the region $x / D=30$ to 80 of interest here was initially checked using a single hot-wire probe. The measured profiles of mean and fluctuating velocities were self-similar, and nearly identical to those reported by Hussein et al. (1994). The momentum integral of the jet computed from these profiles varied by less than $1 \%$ over this range. Further details can be found in Frohnapfel (2003).

\subsection{LDA/hot-wire measurement technique}

The two-point correlation of the streamwise fluctuating velocity along the jet centreline was determined by simultaneously measuring the velocity at a fixed upstream location using an LDA system, and at different downstream locations using a hot-wire anemometry system as shown in figure 1. This novel technique can provide measurements of the streamwise correlation with low statistical uncertainty while avoiding the wake effects associated with measurements using two hot-wires. The LDA measurements were performed using a Dantec fibre-optic LDA system with DANTEC BSA 57N11 processors configured in back-scatter mode with a measuring control volume $30 \mu \mathrm{m}$ in diameter and $300 \mu \mathrm{m}$ long. The flow was seeded using a smoke generator that produced particulates with a diameter of approximately $0.7 \mu \mathrm{m}$. The blower was located inside the enclosure so the air was recirculated to achieve approximately uniform seeding of the jet and ambient air. The hot-wire measurements (HW) were performed using a standard unplated $5 \mu \mathrm{m}$ diameter single-wire probe, with a length-to-diameter ratio of 250. The probe was connected to a DANTEC Mini CTA set up with an overheat of 1.8. The cutoff frequency on the CTA was set higher than the roll-off frequency of the probe. The signal from the hot-wire anemometer was low-pass filtered and then sampled using a IOtech 16-bit A/D board. The hot-wires were calibrated in a second 
Parameter

Integral time scale (ms)

Low-pass filter $(\mathrm{kHz})$

HW sampling frequency $(\mathrm{kHz})$

Total record length (s)

$$
x / D=30.3
$$

3.5

10

40

300

$x / D=43.3$
8.5
10
40
300

$x / D=58.3$

14

3

12

600

TABLE 1. Summary of the sampling times in the LDA/HW measurements.

contoured nozzle jet with a $9.8 \mathrm{~cm}$ diameter outlet before and after each experiment using the approach outlined in George, Beuther \& Shabbir (1989).

The sampling of the signals from the LDA system and the hot-wire anemometry systems were synchronized by connecting the Dantec BSA analyser and the IOtech data acquisition system to the same external clock. The output trigger from the BSA analyser was then used as the external trigger to start the data acquisition system for the hot-wire signal. The LDA system and the IOtech data acquisition system were both set to sample for a fixed length of time once sampling was initiated. The synchronization of the velocity signals measured with the two systems was checked by simultaneously measuring the velocity at essentially the same location using both systems. The resulting velocity traces were in excellent agreement, indicating the signals were properly synchronized.

The effect of the smoke particles on the hot-wire measurements was checked by performing measurements using the hot-wire at the same location with and without the smoke present. There was no visible difference in the character of the velocity signals, and the variance of the fluctuating velocity in the two cases agreed to within experimental uncertainty. Further, any spikes that would occur if impacting particles boiled off would be uncorrelated with the turbulence because their arrival time would be uncorrelated with the turbulence field. Thus they would not affect the correlation computed between the velocities measured with the LDA and HW systems. The effect of the smoke on the hot-wire calibration was also examined by comparing the calibration curves determined before and after the hot-wire was operated for two hours in the seeded jet. The velocities calculated from the two calibration curves differed by less than $2 \%$, a change similar to the drift observed during normal hotwire operation. The results were consistent with the observation that the smoke used did not leave a residue on the wire.

The two-point correlations were measured for cases where the LDA probe was located at $x / D=30.3,43.3$ and 58.3 in a jet with an exit velocity of $50 \mathrm{~m} \mathrm{~s}^{-1}$, corresponding to an exit Reynolds number of approximately 33000 . At each new location, the centreline of the jet was determined using hot-wire anemometry measurements. The LDA probe was focused at this location by moving the focal volume until both beams scattered light due to interference with the hot-wire sensor located on the centreline. The hot-wire was then moved to different positions along the centreline of the jet. In all experiments, the jet was run for approximately 45 minutes after the initial seeding to ensure that the jet and ambient air in the enclosure were uniformly seeded. Measurements were initiated when the sampling rate from the LDA system fell below 600 samples per second and continued until the sampling rate dropped to 150 samples per second due to deposition of the particles. This allowed approximately 30 minutes for measurements. The sampling parameters used at the different locations and the integral time scale at these locations are summarized in table 1. 
Parameter

Number of independent samples $\mathrm{N}$

$\epsilon_{\rho}(\%)$ at $\rho=1$

$\epsilon_{\rho}(\%)$ at $\rho=0.5$

$\epsilon_{\rho}(\%)$ at $\rho=0.1$

$\begin{array}{cc}x / D=30.3 & x / D=43.3 \\ >42500 & >17000 \\ 0.5 & 0.8 \\ 1.0 & 1.5 \\ 4.8 & 7.5\end{array}$

$x / D=58.3$

PIV data

$>21000$
0.7
1.4
6.8

657

3.9

7.8

39

TABLE 2. Summary of uncertainty in the correlations computed from the LDA/HW measurements and the PIV measurements of Fukushima et al. (2000) due to sample size.

\subsection{Data reduction}

The theory for using a burst-mode LDA system in conjunction with a transducer that yields a continuous signal, such as a hot-wire anemometer, was described in detail in George et al. (1978). The residence time-weighted algorithm to compute the cross-correlation between the signals from the two systems is given by

$$
C_{\mathrm{LDA}-\mathrm{HW}}=\frac{\sum_{n=1}^{N} u_{\mathrm{HW}}^{(n)} u_{\mathrm{LDA}}^{(n)} \Delta t^{(n)}}{\sum_{n=1}^{N} \Delta t^{(n)}},
$$

where $\Delta t^{(n)}$ is the residence time of the particle and $u_{\mathrm{HW}}^{(n)}$ and $u_{\mathrm{LDA}}^{(n)}$ are the velocities from the hot-wire and LDA systems, respectively, determined simultaneously whenever a seeding particle enters the LDA scattering volume. The correlations of the HW and LDA signals from the two systems were initially determined by selecting cases when the two velocities were sampled within $10 \mu$ s of each other. The resulting data rate was too low, so instead all of the LDA measurements were used to compute the two-point correlations and the corresponding velocity for the hot-wire system was estimated by linearly interpolating the velocity between the neighbouring times.

\subsection{Statistical accuracy for the LDA/HW and PIV data}

The variability (relative r.m.s. statistical error) in the two-point correlation coefficient is given by (George et al. 1978)

$$
\epsilon_{\rho}=\frac{1}{\sqrt{N}} \frac{1}{\rho},
$$

where $N$ is the number of independent samples and $\rho$ is the correlation coefficient. The absolute error in the correlation coefficient is constant, so the relative error increases as the correlation coefficient decreases. Thus, a large number of independent samples is required to accurately determine the correlation coefficient at large separation distances of particular interest here (because the correlation coefficient is small). Long sampling times were used here to ensure $\epsilon_{\rho}$ was sufficiently small to determine if the correlations at different locations collapsed when they were scaled using similarity variables.

Following George et al. (1978), the number of independent samples in the LDA/HW technique was estimated by $N=T / 2 \tau$, where $T$ is the total length of the record and $\tau$ is the integral time scale. The resulting number of the independent measurements determined from the parameters in table 1 ranged from 17000 to 42500 , and the variability in the coefficient summarized in table 2 was 5 to $8 \%$ when the correlation coefficient was 0.1. The database of PIV measurements performed by Fukushima 


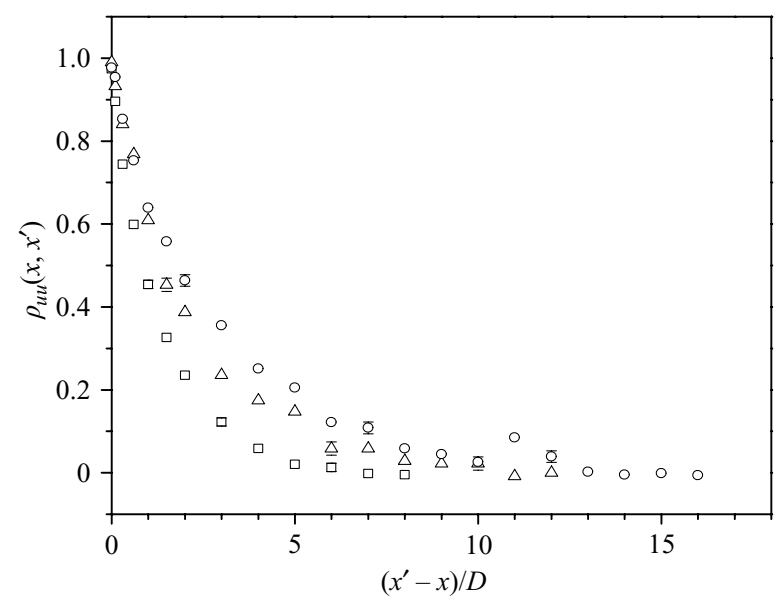

FIGURE 2. The streamwise two-point correlation coefficient of the streamwise fluctuating velocity measured along jet centreline using the LDA/HW technique at $\square, x / D=30.3$; $\triangle, x / D=43.3$; and $\bigcirc, x / D=58.3$. The error bars show the $95 \%$ confidence interval due to the sample size.

et al. (2000) included 657 images, so the uncertainty in the correlation coefficients computed from the PIV measurements (also shown in table 2) were 5 to 10 times larger than those determined using the LDA/HW technique.

\section{Measurements of the streamwise two-point velocity correlations}

The two-point correlation coefficients of the fluctuating streamwise velocity in the streamwise direction given by

$$
\rho_{u u}\left(x, x^{\prime}\right)=\frac{\overline{u(x, t) u^{\prime}\left(x^{\prime}, t\right)}}{\left[\overline{u(x)^{2}} \overline{u^{\prime}\left(x^{\prime}\right)^{2}}\right]^{1 / 2}},
$$

measured along the centreline of the jet with a Reynolds number of 33000 are shown in figure 2. Here and throughout this section, $x$ is the streamwise distance from the nozzle exit. The distance required for the two-point velocity correlation to fall off to a given value increases as the flow evolves downstream, indicating that the characteristic length scale of the large-scale motions is increasing as the flow evolves. The two-point velocity correlations along the centreline presented in the streamwise similarity coordinate are shown in figure 3 . The location of the virtual origin was subtracted from the streamwise position $x$ in determining the similarity coordinate (i.e. $\xi=\ln \left(x-x_{o}\right)$, where $x_{o}$ is the location of the virtual origin). The virtual origin of this jet was $4.6 \mathrm{D}$ downstream of the jet exit. The two-point correlations collapse to within experimental uncertainty over the entire range, indicating that similarity solutions for the two-point velocity correlation do describe the development of the large-scale motions in the streamwise direction of the jet, at least along the centreline.

The correlations computed from the PIV measurements performed by Fukushima et al. (2000) for a jet with a Reynolds number of 2000 show similar trends, although with greater uncertainly (owing to the much smaller number of samples). These data were used to compute the correlation of the streamwise and radial fluctuating velocity both on the centreline and off this axis. Of particular interest are the correlations along lines of constant $\eta=r / \delta$. (Note that this was not reasonable using the LDA/HW 


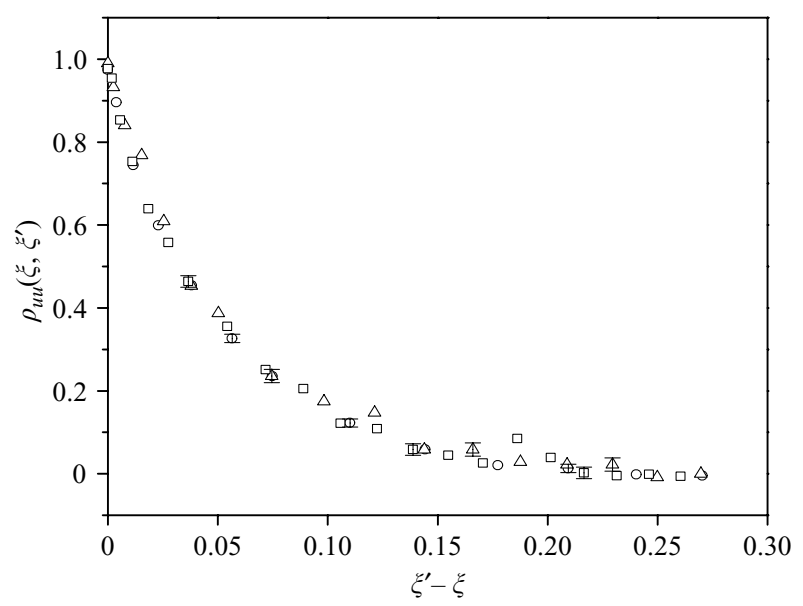

FIGURE 3. The streamwise two-point correlation coefficient of the streamwise fluctuating velocity in similarity coordinates along the jet centreline at $\square, x / D=30.3 ; \triangle, x / D=43.3$; and $\bigcirc, x / D=58.3$. The error bars show the $95 \%$ confidence interval.
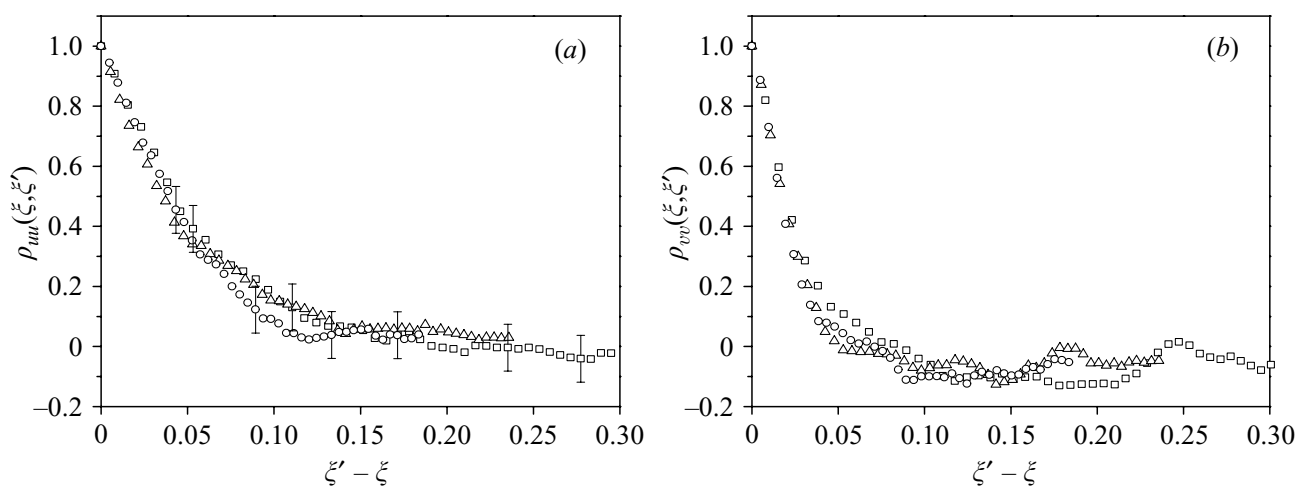

FIGURE 4. The streamwise two-point correlation coefficient of $(a)$ the streamwise fluctuating velocity and $(b)$ the radial fluctuating velocity in similarity coordinates along the jet centreline about $\square, x / D=53 ; \triangle, x / D=72.2$; and $\bigcirc, x / D=80$ computed from the PIV measurements performed by Fukushima et al. (2000). The error bars in $(a)$ show the $95 \%$ confidence interval due to the sampling size in PIV measurements.

technique, since the local turbulence intensity rises rapidly away from the centreline, thereby making the crossflow errors in the HW measurements large.) The two-point correlation coefficients of the streamwise and radial fluctuating velocity along the jet centreline and along a line with $r=\delta(x)$ (where $\delta(x)$ is local half-width of the jet) between $x / D=50$ and $x / D=80$ are shown in the similarity coordinate in figures 4 and 5. The streamwise coordinate $x$ is, again, the distance from the jet exit, while the virtual origin in this jet was $6.75 \mathrm{D}$ downstream of the jet exit. The results for $r=\delta$ are the average of the results from the two sides of the jet on the measurement planes (or positions 180 degrees apart in the azimuthal direction), which may reduce the uncertainty in these results.

The distance over which the radial fluctuating velocity becomes uncorrelated is less than for the streamwise fluctuating velocity at both positions. The correlation coefficients along $r=\delta$ also fall more rapidly than those on the centreline. Overall, the 

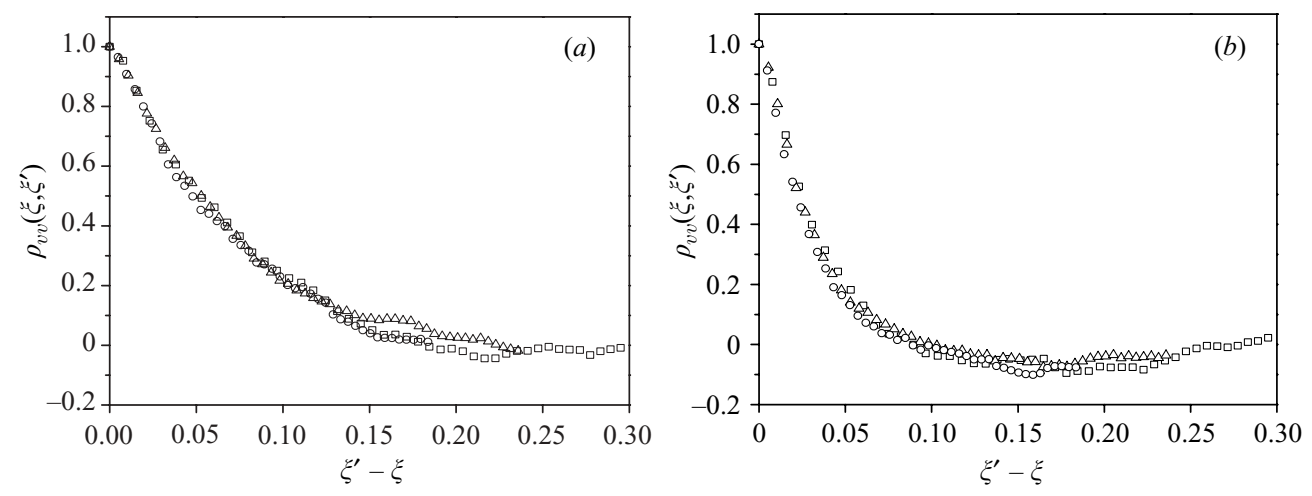

FIGURE 5. The streamwise two-point correlation of $(a)$ the streamwise fluctuating velocity and (b) the radial fluctuating velocity in similarity coordinates along $r / \delta(x)=1$ about $\square, x / D=53$; $\triangle, x / D=72.2$; and $\bigcirc, x / D=80$ from the PIV measurements performed by Fukushima et al. (2000).

two-point velocity correlation coefficients at both radial locations collapse to within experimental uncertainty, suggesting strongly that the two-point similarity solutions deduced here do describe the growth of the structures in the streamwise direction. Interestingly, the correlation along the centreline of the jet with Reynolds number of 2000 in similarity variables initially falls off more slowly than result for the higherReynolds-number jet, indicating that the structures in the jets may depend on the Reynolds number of the jet as predicted in the analysis.

\section{Summary and conclusions}

It was shown that the governing equations for the two-point velocity correlations in the far field of the axisymmetric jet admit equilibrium similarity solutions for a finite-Reynolds-number jet, where the two-point correlations were written as the product of a scale dependent on the streamwise position of the two points relative to the jet origin and a function dependent only on similarity variables. Physically, this implies that the turbulent processes at the different scales of motion in the jet are in equilibrium when the jet is evolving in a manner consistent with the proposed equilibrium similarity solution. The two-point similarity solutions deduced here are more general than those deduced by analysing the equations for the single-point moments (Hussein et al. 1994) because they include information about how the energy is contained in and transferred among the different scales of motion that is not contained in the single-point equations.

Further, like the analysis of the single-point moments in a range of flows (George 1995; Moser et al. 1998), it was shown that the governing equations for the equilibrium similarity solutions could not be reduced to a form independent of the jet growth rate. Thus the results indicate that there is not a universal solution that describes the large-scale structures in the far field of the round jet, so that the structures present may depend on how the flow is generated. It cannot be determined from the analysis whether the round jet generated from different sources would evolve to different solutions, but the investigations of Boersma et al. (1998) and Mi et al. (2001) show such a dependence.

Gamard et al. $(2002,2004)$ showed that the two-point velocity correlations with separation distances in the radial and azimuthal direction measured on different planes 
in the fully developed region of the round jet collapsed when they were scaled in a manner consistent with the analysis outlined here. The correlations in the streamwise direction were determined here from combined LDA/HW measurements in a jet with a Reynolds number of 33000 and from PIV measurements in a jet with a Reynolds number of 2000 performed by Fukushima et al. (2000). The streamwise velocity correlations in the individual jets collapsed when they were scaled in the manner predicted in the analysis. Thus the equilibrium similarity solutions deduced here do appear to describe the evolution of the turbulent motions in the far field of the round jet. The results in the two jets examined here did differ, indicating that the structures in the jet may depend on the Reynolds number of the jet as predicted by the analysis.

The equilibrium similarity solutions for the two-point velocity correlations have implications on attempts to model the pressure-strain, the dissipation, the mean square vorticity, and attempts to represent the turbulent motions using techniques such as the proper orthogonal decomposition, all of which require two-point information. For example, it can be shown that the turbulence dissipation and mean square vorticity have similarity solutions when the two-point velocity correlations have similarity solutions as discussed in Appendix B. The similarity solution for the two-point velocity correlations also only depends on the separation distance between the points in the logarithmic streamwise similarity variable, so the correlation is statistically homogeneous in this direction. Since the similarity variable in the streamwise direction is the logarithm of the physical coordinate from the jet virtual origin, the semi-infinite physical coordinate is also transformed into an infinite similarity coordinate $(-\infty, \infty)$. As a result, the optimal modes to represent the energy of the turbulent motions in the streamwise direction are Fourier modes in the similarity coordinate system. The consequences of this result will be explored in subsequent work.

Earlier versions of this analysis were presented in the $\mathrm{PhD}$ dissertation of Ewing (1995) and in Ewing \& George (1995), and formed the basis of the invited lecture by WKG at the 2003 APS/DFD Annual meeting. The HW/LDA experiments here were part of the Masters thesis of Frohnapfel (2003). The authors would like to thank L. Jernquist, T. G. Johansson, and P. B. V. Johansson for their assistance in setting up and performing the experiments presented in this work. The work was originally supported in part by funding from the National Science Foundation of the United States of America. Subsequently the work of WKG, BF and JP was supported by Vetenskapsrådet, the Swedish Research Foundation, while that of DE was supported by the Natural Sciences and Engineering Research Council of Canada.

\section{Appendix A. Equation for two-point velocity correlations}

$$
\begin{aligned}
& {\left[U_{s}\left(x_{1}\right) \frac{\partial Q_{s}^{(i, j)}}{\partial x_{1}}\right] f(\eta) q_{i, j}+\left[Q_{s}^{(i, j)} U_{s}\left(x_{1}\right) \frac{\partial v}{\partial x_{1}}\right] f(\eta) \frac{\partial q_{i, j}}{\partial v}-\left(\left[Q_{s}^{(i, j)} \frac{\mathrm{d} U_{s}\left(x_{1}\right)}{\mathrm{d} x_{1}}\right]\right.} \\
& \left.+\left[2 \frac{Q_{s}^{(i, j)} U_{s}\left(x_{1}\right)}{\delta\left(x_{1}\right)} \frac{\mathrm{d} \delta}{\mathrm{d} x_{1}}\right]\right)\left(\frac{\partial q_{i, j}}{\partial \eta}+\frac{q_{3, j}}{\eta} \delta_{i 3}\right) \frac{1}{\eta} \int_{0}^{\eta} \tilde{\eta} f(\tilde{\eta}) \mathrm{d} \tilde{\eta} \\
& +\left[\frac{Q_{s}^{(i, j)} U_{s}\left(x_{1}\right)}{\delta\left(x_{1}\right)} \frac{\mathrm{d} \delta}{\mathrm{d} x_{1}}\right] f(\eta) q_{i, 3} \delta_{j 3}+\left[U_{s}\left(x_{1}^{\prime}\right) \frac{\partial Q_{s}^{(i, j)}}{\partial x_{1}^{\prime}}\right] f\left(\eta^{\prime}\right) q_{i, j} \\
& +\left[Q_{s}^{(i, j)} U_{s}\left(x_{1}^{\prime}\right) \frac{\partial v}{\partial x_{1}^{\prime}}\right] f\left(\eta^{\prime}\right) \frac{\partial q_{i, j}}{\partial v}-\left(\left[Q_{s}^{(i, j)} \frac{\mathrm{d} U_{s}\left(x_{1}^{\prime}\right)}{\mathrm{d} x_{1}^{\prime}}\right]+\left[2 \frac{Q_{s}^{(i, j)} U_{s}\left(x_{1}^{\prime}\right)}{\delta\left(x_{1}^{\prime}\right)} \frac{\mathrm{d} \delta}{\mathrm{d} x_{1}^{\prime}}\right]\right)
\end{aligned}
$$




$$
\begin{aligned}
& \times\left(\frac{\partial q_{i, j}}{\partial \eta^{\prime}}+\frac{q_{i, 3}}{\eta^{\prime}} \delta_{j 3}\right) \frac{1}{\eta^{\prime}} \int_{0}^{\eta^{\prime}} \tilde{\eta} f(\tilde{\eta}) \mathrm{d} \tilde{\eta}+\left[\frac{Q_{s}^{(i, j)} U_{s}\left(x_{1}^{\prime}\right)}{\delta\left(x_{1}^{\prime}\right)} \frac{\mathrm{d} \delta}{\mathrm{d} x_{1}^{\prime}}\right] f\left(\eta^{\prime}\right) q_{3, j} \delta_{i 3} \\
& =-\frac{1}{\rho}\left\{\left[\frac{\partial \Pi_{s, 1}^{(, j)}}{\partial x_{1}}\right] \pi_{j}^{1} \delta_{i 1}+\left[\Pi_{s, 1}^{(, j)} \frac{\partial v}{\partial x_{1}}\right] \frac{\partial \pi_{j}^{1}}{\partial v} \delta_{i 1}-\left[\frac{\Pi_{s, 1}^{(, j)}}{\delta\left(x_{1}\right)} \frac{\mathrm{d} \delta}{\mathrm{d} x_{1}}\right]\left(\eta \frac{\partial \pi_{j}^{1}}{\partial \eta}\right) \delta_{i 1}\right. \\
& +\left[\frac{\partial \Pi_{s, 2}^{(i,)}}{\partial x_{1}^{\prime}}\right] \pi_{i}^{2} \delta_{j 1}+\left[\Pi_{s, 1}^{(, j)} \frac{\partial v}{\partial x_{1}^{\prime}}\right] \frac{\partial \pi_{i}^{2}}{\partial v} \delta_{j 1}-\left[\frac{\Pi_{s, 1}^{(i,)}}{\delta\left(x_{1}^{\prime}\right)} \frac{\mathrm{d} \delta}{\mathrm{d} x_{1}^{\prime}}\right] \eta^{\prime} \frac{\partial \pi_{i}^{2}}{\partial \eta^{\prime}} \delta_{j 1}+\left[\frac{\Pi_{s, 1}^{(, j)}}{\delta\left(x_{1}\right)}\right] \\
& \left.\times\left(\frac{\partial \pi_{j}^{1}}{\partial \eta} \delta_{i 2}-\frac{1}{\eta} \frac{\partial \pi_{j}^{1}}{\partial \theta} \delta_{i 3}\right)+\left[\frac{\Pi_{s, 2}^{(i,)}}{\delta\left(x_{1}^{\prime}\right)}\right]\left(\frac{\partial \pi_{i}^{2}}{\partial \eta^{\prime}} \delta_{j 2}+\frac{1}{\eta^{\prime}} \frac{\partial \pi_{i}^{2}}{\partial \theta} \delta_{j 3}\right)\right\}-\left[\frac{\partial T_{s, 1}^{(1 i, j)}}{\partial x_{1}}\right] t_{1 i, j}^{1} \\
& -\left[T_{s, 1}^{(1 i, j)} \frac{\partial v}{\partial x_{1}}\right] \frac{\partial t_{1 i, j}^{1}}{\partial v}+\left[\frac{T_{s, 1}^{(1 i, j)}}{\delta\left(x_{1}\right)} \frac{\mathrm{d} \delta}{\mathrm{d} x_{1}}\right] \eta \frac{\partial t_{1 i, j}^{1}}{\partial \eta}-\left[\frac{T_{s, 1}^{(2 i, j)}}{\delta\left(x_{1}\right)}\right] \frac{1}{\eta} \frac{\partial \eta t_{2 i, j}^{1}}{\partial \eta} \\
& +\left[\frac{T_{s, 1}^{(3 i, j)}}{\delta\left(x_{1}\right)}\right] \frac{1}{\eta} \frac{\partial t_{3 i, j}^{1}}{\partial \theta}+\left[\frac{T_{s, 1}^{(33, j)}}{\delta\left(x_{1}\right)}\right] \frac{t_{33, j}^{1}}{\eta} \delta_{i 2}-\left[\frac{T_{s, 1}^{(23, j)}}{\delta\left(x_{1}\right)}\right] \frac{t_{23, j}^{1}}{\eta} \delta_{i 3}-\left[\frac{\partial T_{s, 2}^{(i, 1 j)}}{\partial x_{1}^{\prime}}\right] t_{i, 1 j}^{2} \\
& -\left[T_{s, 2}^{(i, 1 j)} \frac{\partial v}{\partial x_{1}^{\prime}}\right] \frac{\partial t_{i, 1 j}^{2}}{\partial v}+\left[\frac{T_{s, 2}^{(i, 1 j)}}{\delta\left(x_{1}^{\prime}\right)} \frac{\mathrm{d} \delta}{\mathrm{d} x_{1}^{\prime}}\right] \eta^{\prime} \frac{\partial t_{i, 1 j}^{2}}{\partial \eta^{\prime}}-\left[\frac{T_{s, 2}^{(i, 2 j)}}{\delta\left(x_{1}^{\prime}\right)}\right] \frac{1}{\eta^{\prime}} \frac{\partial \eta^{\prime} t_{i, 2 j}^{2}}{\partial \eta^{\prime}}-\left[\frac{T_{s, 2}^{(i, 3 j)}}{\delta\left(x_{1}^{\prime}\right)}\right] \\
& \times \frac{1}{\eta^{\prime}} \frac{\partial t_{i, 3 j}^{2}}{\partial \theta}+\left[\frac{T_{s, 2}^{(i, 33)}}{\delta\left(x_{1}^{\prime}\right)}\right] \frac{t_{i, 33}^{2}}{\eta^{\prime}} \delta_{j 2}-\left[\frac{T_{s, 2}^{(i, 23)}}{\delta\left(x_{1}^{\prime}\right)}\right] \frac{t_{i, 23}^{2}}{\eta^{\prime}} \delta_{j 3}-\left\{\left[Q_{s}^{(1, j)} \frac{\mathrm{d} U_{s}}{\mathrm{~d} x_{1}}\right] q_{1, j} f(\eta)\right. \\
& \left.-\left[\frac{Q_{s}^{(1, j)} U_{s}\left(x_{1}\right)}{\delta\left(x_{1}\right)} \frac{\mathrm{d} \delta}{\mathrm{d} x_{1}}\right] q_{1, j} \eta \frac{\mathrm{d} f}{\mathrm{~d} \eta}+\left[\frac{Q_{s}^{(2, j)} U_{s}\left(x_{1}\right)}{\delta\left(x_{1}\right)}\right] q_{2, j} \frac{\mathrm{d} f}{\mathrm{~d} \eta}\right\} \delta_{i 1} \\
& -\left\{\left(-4\left[Q_{s}^{(1, j)} \frac{\mathrm{d} \delta}{\mathrm{d} x_{1}} \frac{\mathrm{d} U_{s}}{\mathrm{~d} x_{1}}\right]-\left[Q_{s}^{(1, j)} \delta \frac{\mathrm{d}^{2} U_{s}}{\mathrm{~d} x_{1}^{2}}\right]-2\left[Q_{s}^{(1, j)} U_{s}\left(x_{1}\right) \frac{\mathrm{d}^{2} \delta}{\mathrm{d} x_{1}^{2}}\right]\right.\right. \\
& \left.-2\left[Q_{s}^{(1, j)} \frac{U_{s}\left(x_{1}\right)}{\delta\left(x_{1}\right)}\left(\frac{\mathrm{d} \delta}{\mathrm{d} x_{1}}\right)^{2}\right]\right) q_{1, j} \frac{1}{\eta} \int_{0}^{\eta} \tilde{\eta} f \mathrm{~d} \tilde{\eta}+\left(2\left[Q_{s}^{(1, j)} \frac{\mathrm{d} U_{s}}{\mathrm{~d} x_{1}} \frac{\mathrm{d} \delta}{\mathrm{d} x_{1}}\right]\right. \\
& \left.+2\left[\frac{Q_{s}^{(1, j)} U_{s}\left(x_{1}\right)}{\delta}\left(\frac{\mathrm{d} \delta}{\mathrm{d} x_{1}}\right)^{2}\right]+\left[Q_{s}^{(1, j)} U_{s}\left(x_{1}\right) \frac{\mathrm{d}^{2} \delta}{\mathrm{d} x_{1}^{2}}\right]\right) \eta q_{1, j} f(\eta) \\
& -\left[Q_{s}^{(1, j)} \frac{U_{s}\left(x_{1}\right)}{\delta\left(x_{1}\right)}\left(\frac{\mathrm{d} \delta}{\mathrm{d} x_{1}}\right)^{2}\right] q_{1, j} \eta \frac{\mathrm{d} \eta f(\eta)}{\mathrm{d} \eta}+\left(\left[Q_{s}^{(2, j)} \frac{\mathrm{d} U_{s}}{\mathrm{~d} x_{1}}\right]+2\left[Q_{s}^{(2, j)} \frac{U_{s}\left(x_{1}\right)}{\delta\left(x_{1}\right)} \frac{\mathrm{d} \delta}{\mathrm{d} x_{1}}\right]\right) \\
& \left.\times\left(q_{2, j} \frac{1}{\eta^{2}} \int_{0}^{\eta} \tilde{\eta} f \mathrm{~d} \tilde{\eta}-f(\eta)\right)+\left[Q_{s}^{(2, j)} \frac{U_{s}\left(x_{1}\right)}{\delta\left(x_{1}\right)} \frac{\mathrm{d} \delta}{\mathrm{d} x_{1}}\right] q_{2, j} \frac{\mathrm{d} \eta f}{\mathrm{~d} \eta}\right\} \delta_{i 2} \\
& -\left\{\left[Q_{s}^{(i, 1)} \frac{\mathrm{d} U_{s}}{\mathrm{~d} x_{1}^{\prime}}\right] q_{i, 1} f\left(\eta^{\prime}\right)-\left[\frac{Q_{s}^{(i, 1)} U_{s}\left(x_{1}^{\prime}\right)}{\delta\left(x_{1}^{\prime}\right)} \frac{\mathrm{d} \delta}{\mathrm{d} x_{1}^{\prime}}\right] q_{i, 1} \eta^{\prime} \frac{\mathrm{d} f}{\mathrm{~d} \eta^{\prime}}+\left[\frac{Q_{s}^{(i, 2)} U_{s}\left(x_{1}^{\prime}\right)}{\delta\left(x_{1}^{\prime}\right)}\right]\right. \\
& \left.\times q_{i, 2} \frac{\mathrm{d} f}{\mathrm{~d} \eta^{\prime}}\right\} \delta_{j 1}-\left\{\left(-4\left[Q_{s}^{(i, 1)} \frac{\mathrm{d} \delta}{\mathrm{d} x_{1}^{\prime}} \frac{\mathrm{d} U_{s}}{\mathrm{~d} x_{1}^{\prime}}\right]-\left[Q_{s}^{(i, 1)} \delta\left(x_{1}^{\prime}\right) \frac{\mathrm{d}^{2} U_{s}}{\mathrm{~d} x_{1}^{\prime 2}}\right]\right.\right. \\
& \left.-2\left[Q_{s}^{(i, 1)} U_{s}\left(x_{1}^{\prime}\right) \frac{\mathrm{d}^{2} \delta}{\mathrm{d} x_{1}^{\prime 2}}\right]-2\left[Q_{s}^{(i, 1)} \frac{U_{s}\left(x_{1}^{\prime}\right)}{\delta\left(x_{1}^{\prime}\right)}\left(\frac{\mathrm{d} \delta}{\mathrm{d} x_{1}^{\prime}}\right)^{2}\right]\right) q_{i, 1} \frac{1}{\eta^{\prime}} \int_{0}^{\eta^{\prime}} \tilde{\eta} f \mathrm{~d} \tilde{\eta}
\end{aligned}
$$




$$
\begin{aligned}
& +\left(2\left[Q_{s}^{(i, 1)} \frac{\mathrm{d} U_{s}}{\mathrm{~d} x_{1}^{\prime}} \frac{\mathrm{d} \delta}{\mathrm{d} x_{1}^{\prime}}\right]+2\left[\frac{Q_{s}^{(i, 1)} U_{s}\left(x_{1}^{\prime}\right)}{\delta\left(x_{1}^{\prime}\right)}\left(\frac{\mathrm{d} \delta}{\mathrm{d} x_{1}^{\prime}}\right)^{2}\right]+\left[Q_{s}^{(i, 1)} U_{s}\left(x_{1}^{\prime}\right) \frac{\mathrm{d}^{2} \delta}{\mathrm{d} x_{1}^{\prime 2}}\right]\right) \\
& \times \eta^{\prime} q_{i, 1} f\left(\eta^{\prime}\right)-\left[Q_{s}^{(i, 1)} \frac{U_{s}\left(x_{1}^{\prime}\right)}{\delta\left(x_{1}^{\prime}\right)}\left(\frac{\mathrm{d} \delta}{\mathrm{d} x_{1}^{\prime}}\right)^{2}\right] q_{i, 1} \frac{\mathrm{d} \eta^{\prime} f\left(\eta^{\prime}\right)}{\mathrm{d} \eta^{\prime}}+\left(\left[Q_{s}^{(i, 2)} \frac{\mathrm{d} U_{s}}{\mathrm{~d} x_{1}^{\prime}}\right]\right. \\
& \left.+2\left[Q_{s}^{(i, 2)} \frac{U_{s}\left(x_{1}^{\prime}\right)}{\delta\left(x_{1}^{\prime}\right)} \frac{\mathrm{d} \delta}{\mathrm{d} x_{1}^{\prime}}\right]\right)\left(q_{i, 2} \frac{1}{\eta^{\prime 2}} \int_{0}^{\eta^{\prime}} \tilde{\eta}^{\prime} f \mathrm{~d} \tilde{\eta}-f\left(\eta^{\prime}\right)\right)+\left[Q_{s}^{(i, 2)} \frac{U_{s}\left(x_{1}^{\prime}\right)}{\delta\left(x_{1}^{\prime}\right)} \frac{\mathrm{d} \delta}{\mathrm{d} x_{1}^{\prime}}\right] \\
& \left.\times q_{i, 2} \frac{\mathrm{d} \eta^{\prime} f}{\mathrm{~d} \eta^{\prime}}\right\} \delta_{j 2}+v\left\{\left[\frac{\partial^{2} Q_{s}^{(i, j)}}{\partial x_{1}^{2}}\right] q_{i, j}+\left(\left[\frac{Q_{s}^{(i, j)}}{\delta\left(x_{1}\right)^{2}}\left(\frac{\mathrm{d} \delta}{\mathrm{d} x_{1}}\right)^{2}\right]-\left[\frac{Q_{s}^{(i, j)}}{\delta\left(x_{1}\right)} \frac{\mathrm{d}^{2} \delta}{\mathrm{d} x_{1}^{2}}\right]\right.\right. \\
& \left.-\left[\frac{2}{\delta\left(x_{1}\right)} \frac{\mathrm{d} \delta}{\mathrm{d} x_{1}} \frac{\partial Q_{s}^{(i, j)}}{\partial x_{1}}\right]\right) \eta \frac{\partial q_{i, j}}{\partial \eta}+\left(2\left[\frac{\partial v}{\partial x_{1}} \frac{\partial Q_{s}^{(i, j)}}{\partial x_{1}}\right]+\left[Q_{s}^{(i, j)} \frac{\partial^{2} v}{\partial x_{1}^{2}}\right]\right) \frac{\partial q_{i, j}}{\partial v} \\
& +\left[Q_{s}^{(i, j)}\left(\frac{\partial v}{\partial x_{1}}\right)^{2}\right] \frac{\partial^{2} q_{i, i}}{\partial v^{2}}-2\left[\frac{Q_{s}^{(i, j)}}{\delta} \frac{\mathrm{d} \delta}{\mathrm{d} x_{1}} \frac{\partial v}{\partial x_{1}}\right] \eta \frac{\partial^{2} q_{i, j}}{\partial \eta \partial v}+\left[\frac{Q_{s}^{(i, j)}}{\delta\left(x_{1}\right)^{2}}\left(\frac{\mathrm{d} \delta}{\mathrm{d} x_{1}}\right)^{2}\right] \\
& \left.\times \eta \frac{\partial}{\partial \eta} \eta \frac{\partial q_{i, j}}{\partial \eta}+\left[\frac{Q_{s}^{(i, j)}}{\delta\left(x_{1}\right)^{2}}\right]\left(\frac{1}{\eta} \frac{\partial}{\partial \eta} \eta \frac{\partial q_{i, j}}{\partial \eta}+\frac{1}{\eta^{2}} \frac{\partial^{2} q_{i, j}}{\partial \theta^{2}}\right)\right\}-v\left\{\left[\frac{Q_{s}^{(2, j)}}{\delta^{2}\left(x_{1}\right)}\right] \frac{q_{2, j}}{\eta^{2}}\right. \\
& \left.-\left[\frac{2 Q_{s}^{(3, j)}}{\delta^{2}\left(x_{1}\right)}\right] \frac{1}{\eta^{2}} \frac{\partial q_{3, j}}{\partial \theta}\right\} \delta_{i 2}-v\left\{\left[\frac{Q_{s}^{(3, j)}}{\delta^{2}\left(x_{1}\right)}\right] \frac{q_{3, j}}{\eta^{2}}+\left[\frac{2 Q_{s}^{(2, j)}}{\delta^{2}\left(x_{1}\right)}\right] \frac{1}{\eta^{2}} \frac{\partial q_{2, j}}{\partial \theta}\right\} \delta_{i 3} \\
& +v\left\{\left[\frac{\partial^{2} Q_{s}^{(i, j)}}{\partial x_{1}^{\prime 2}}\right] q_{i, j}+\left(\left[\frac{Q_{s}^{(i, j)}}{\delta\left(x_{1}^{\prime}\right)^{2}}\left(\frac{\mathrm{d} \delta}{\mathrm{d} x_{1}^{\prime}}\right)^{2}\right]+\left[\frac{Q_{s}^{(i, j)}}{\delta\left(x_{1}^{\prime}\right)} \frac{\mathrm{d}^{2} \delta}{\mathrm{d} x_{1}^{\prime 2}}\right]\right.\right. \\
& \left.-\left[\frac{2}{\delta\left(x_{1}^{\prime}\right)} \frac{\mathrm{d} \delta}{\mathrm{d} x_{1}^{\prime}} \frac{\partial Q_{s}^{(i, j)}}{\partial x_{1}^{\prime}}\right]\right) \eta^{\prime} \frac{\partial q_{i, j}}{\partial \eta^{\prime}}+\left(2\left[\frac{\partial v}{\partial x_{1}^{\prime}} \frac{\partial Q_{s}^{(i, j)}}{\partial x_{1}^{\prime}}\right]+\left[Q_{s}^{(i, j)} \frac{\partial^{2} v}{\partial x_{1}^{\prime 2}}\right]\right) \frac{\partial q_{i, j}}{\partial v} \\
& +\left(\left[Q_{s}^{(i, j)}\left(\frac{\partial v}{\partial x_{1}^{\prime}}\right)^{2}\right]-2\left[\frac{Q_{s}^{(i, j)}}{\delta\left(x_{1}^{\prime}\right)} \frac{\mathrm{d} \delta}{\mathrm{d} x_{1}^{\prime}} \frac{\partial v}{\partial x_{1}^{\prime}}\right]\right) \eta^{\prime} \frac{\partial^{2} q_{i, j}}{\partial \eta^{\prime} \partial v}+\left[\frac{Q_{s}^{(i, j)}}{\delta\left(x_{1}^{\prime}\right)^{2}}\left(\frac{\mathrm{d} \delta}{\mathrm{d} x_{1}^{\prime}}\right)^{2}\right] \\
& \left.\times \eta^{\prime} \frac{\partial}{\partial \eta^{\prime}} \eta^{\prime} \frac{\partial q_{i, j}}{\partial \eta^{\prime}}+\left[\frac{Q_{s}^{(i, j)}}{\delta\left(x_{1}^{\prime}\right)^{2}}\right]\left(\frac{1}{\eta^{\prime}} \frac{\partial}{\partial \eta^{\prime}} \eta^{\prime} \frac{\partial q_{i, j}}{\partial \eta^{\prime}}+\frac{1}{\eta^{\prime 2}} \frac{\partial^{2} q_{i, j}}{\partial \theta^{2}}\right)\right\}-v\left\{\left[\frac{Q_{s}^{(i, 2)}}{\delta^{2}\left(x_{1}^{\prime}\right)}\right] \frac{q_{i, 2}}{\eta^{\prime 2}}\right. \\
& \left.+\left[\frac{2 Q_{s}^{(i, 3)}}{\delta^{2}\left(x_{1}^{\prime}\right)}\right] \frac{1}{\eta^{\prime 2}} \frac{\partial q_{i, 3}}{\partial \theta}\right\} \delta_{j 2}-v\left\{\left[\frac{Q_{s}^{(i, 3)}}{\delta^{2}\left(x_{1}^{\prime}\right)}\right] \frac{q_{i, 3}}{\eta^{\prime 2}}-\left[\frac{2 Q_{s}^{(i, 2)}}{\delta^{2}\left(x_{1}^{\prime}\right)}\right] \frac{1}{\eta^{\prime 2}} \frac{\partial q_{i, 2}}{\partial \theta}\right\} \delta_{j 3} .
\end{aligned}
$$

\section{Appendix B. Similarity of the velocity derivative correlations}

Similarity solutions for the dissipation of the turbulent kinetic energy, $\epsilon$, given by

$$
\epsilon=\lim _{x_{k} \rightarrow x_{k}^{\prime}} 2 v \overline{e_{i j} e_{i j}},
$$

where $e_{i j}$ is the rate-of-strain tensor given by (see Batchelor 1967)

$$
e_{i j}=\frac{1}{2}\left(\frac{1}{h^{(j)}} \frac{\partial u_{i}}{\partial x_{j}}+\frac{1}{h^{(i)}} \frac{\partial u_{j}}{\partial x_{i}}\right)+\frac{u_{2}}{x_{2}} \delta_{j 3} \delta_{i 3}-\frac{1}{2} \frac{u_{3}}{x_{2}}\left[\delta_{i 2} \delta_{j 3}+\delta_{i 3} \delta_{j 2}\right],
$$


or the two-point correlation for the vorticity given by

$$
\begin{aligned}
\overline{\omega_{i} \omega_{l}^{\prime}}=\frac{1}{4} \epsilon_{i j k} \epsilon_{l m n}\left\{\frac{1}{h^{(j)} h^{(m)}} \frac{\partial^{2} R_{k, n}}{\partial x_{j} \partial x_{m}^{\prime}}+\frac{1}{x_{2}} \frac{1}{h^{(m)}} \frac{\partial R_{3, n}}{\partial x_{m}^{\prime}} \delta_{k 3} \delta_{j 2}\right. \\
\left.+\frac{1}{x_{2}^{\prime}} \frac{1}{h^{(j)}} \frac{\partial R_{k, 3}}{\partial x_{j}} \delta_{n 3} \delta_{m 2}+\frac{R_{3,3}}{x_{2} x_{2}^{\prime}} \delta_{k 3} \delta_{j 2} \delta_{n 3} \delta_{m 2}\right\},
\end{aligned}
$$

where $\epsilon_{i j k}$ is the alternating tensor, can be deduced by recognizing that (cf. Hinze 1975)

$$
\overline{\frac{\partial u_{i}}{\partial x_{k}} \frac{\partial u_{j}^{\prime}}{\partial x_{l}^{\prime}}}=\frac{\partial^{2}}{\partial x_{k} \partial x_{l}^{\prime}}\left(\overline{u_{i} u_{j}^{\prime}}\right),
$$

since $u_{i}$ is not a function of $x_{i}^{\prime}$ nor $u_{j}^{\prime}$ a function of $x_{i}$. Thus, similarity solutions for the two-point velocity gradients can be used to deduce similarity solutions for the two-point velocity correlations. For example,

$$
\begin{array}{r}
\frac{\partial^{2} R_{k, n}}{\partial x_{1} \partial x_{1}^{\prime}}=\left[\frac{Q^{(k, n)}}{x_{1} x_{1}^{\prime}}\right]\left\{q_{k, n}+\eta \frac{\partial}{\partial \eta}+\eta^{\prime} \frac{\partial}{\partial \eta^{\prime}}+\eta \eta^{\prime} \frac{\partial^{2}}{\partial \eta \partial \eta^{\prime}}\right. \\
+\eta^{\prime} \frac{\partial^{2}}{\partial \eta^{\prime} \partial v} \\
\left.-\eta \frac{\partial^{2}}{\partial \eta \partial v}-\frac{\partial^{2}}{\partial v^{2}}\right\} q_{k, n}
\end{array}
$$

or

$$
\frac{\partial^{2} R_{k, n}}{\partial x_{2} \partial x_{2}^{\prime}}=\left[\frac{Q^{(k, n)}}{\delta\left(x_{1}\right) \delta\left(x_{1}^{\prime}\right)}\right] \frac{\partial^{2} q_{k, n}}{\partial \eta \partial \eta^{\prime}} .
$$

This can be done for all the terms in (B 1) and (B 3) and it can be shown that the scale for all the terms is proportional to

$$
\left[\frac{U_{s}\left(x_{1}\right) U_{s}\left(x_{1}^{\prime}\right)}{\delta\left(x_{1}\right) \delta\left(x_{1}^{\prime}\right)}\right]
$$

It follows that the dissipation of kinetic energy per unit mass can be written as

$$
\epsilon=\lim _{x_{k} \rightarrow x_{k}^{\prime}}\left[2 v \frac{U_{s}\left(x_{1}\right) U_{s}\left(x_{1}^{\prime}\right)}{\delta\left(x_{1}\right) \delta\left(x_{1}^{\prime}\right)}\right] D\left(\eta, \eta^{\prime}, \theta, v, *\right)=\left[2 v \frac{U_{s}^{2}\left(x_{1}\right)}{\delta^{2}\left(x_{1}\right)}\right] D(\eta, *),
$$

consistent with the similarity solution for the dissipation assumed in the single-point analysis (e.g. Hussein et al. 1994). The two-point vorticity correlation tensor can be written as

$$
\overline{\omega_{i} \omega_{l}^{\prime}}=\left[\frac{U_{s}\left(x_{1}\right) U_{s}\left(x_{1}^{\prime}\right)}{\delta\left(x_{1}\right) \delta\left(x_{1}^{\prime}\right)}\right] h_{i, l}\left(\eta, \eta^{\prime}, \theta, v, *\right)
$$

when the two-point velocity correlation tensor has a similarity solution. This reduces to a similarity solution for the single-point vorticity moments in the limit of zero separation distance.

\section{REFERENCES}

BAtChEloR, G. K. 1948 Energy decay and self-preserving correlation functions in isotropic turbulence. Q. Appl. Math. 6, 97-116.

Batchelor, G. K. 1967 An Introduction to Fluid Dynamics. Cambridge University Press.

Batchelor, G. K. \& Gill, E. A. 1962 Analysis of the instability of axisymmetric jets. J. Fluid Mech. 14, 529-551.

Boersma, B. J., Brethouwer, G. \& Nieuwstadt, F. T. M. 1998 A numerical investigation of the effect of inflow conditions on the self-similar region of a round jet. Phys. Fluids 10, 899-909. 
Burattini, P., Antonia, R. A. \& Danaila, L. 2005 Similarity in the far field of a turbulent round jet. Phys. Fluids 17, 025101.

Dimotakis, P. E., Maike-Lye, R. C. \& Papantoniou, D. A. 1983 Structure and dynamics of round turbulent jets. Phys. Fluids 26, 3185-3192.

EwING, D. 1995 On multi-point similarity solutions in turbulent free-shear flows. PhD thesis, State University of New York at Buffalo.

EwING, D. \& GEORGE, W. K. 1995 Similarity analysis of the two-point velocity correlation tensor in a turbulent axisymmetric jet. In Turbulence, Heat, and Mass Transfer I (ed. K. Hanjalic \& J. C. P. Pereira), pp. 49-56. Begell House.

Ewing, D., George, W. K., Rogers, M. M. \& Moser, R. D. 2007 Two-point similarity in temporally evolving plane wakes. J. Fluid Mech. 577, 287-307.

Freund, J. B. \& Colonius, T. 2002 POD analysis of sound generation by a turbulent jet. AIAA Paper 2002-0072.

Frohnapfel, B. 2003 Multi-point similarity of the axisymmetric turbulent far jet and its implication for the POD. Master's thesis, Chalmers University of Technology/Friedrich-AlexanderUniversity at Erlangen-Nuremberg, Gothenburg, Sweden and Erlangen,Germany.

Fukushima, C., Aanen, L. \& Westerweel, J. 2000 Investigation of the mixing process in an axisymmetric turbulent jet using PIV and LIF. Proc. 10th International Symposium on Applications of Laser Techniques to Fluid Mechanics (Lisbon, Portugal).

Gamard, S., George, W. K., Jung, D. \& Woodward, S. 2002 Application of a slice POD to the far field of an axisymmetric turbulent jet. Phys. Fluids 14, 2515-2522.

Gamard, S., Jung, D. \& George, W. K. 2004 Downstream evolution of the most energetic modes in a turbulent axisymmetric jet at high Reynolds number. Part 2. The far-field region. J. Fluid Mech. 514, 205-230.

George, W. K. 1989 The self-preservation of turbulent flows and its relation to initial conditions and coherent structures. In Advances in Turbulence (ed. W. K. George \& R. E. Arndt), pp. 39-73. Hemisphere.

George, W. K. 1992 Decay of homogeneous isotropic turbulence. Phys. Fluids 4, 1492-1508.

George, W. K. 1995 Some new ideas for similarity of turbulent shear flows. In Turbulence, Heat, and Mass Transfer I (ed. K. Hanjalic \& J. C. F. Pereira), pp. 13-24. Begell House.

George, W. K., Beuther, P. D. \& Lumley, J. L. 1978 Processing of random signals. In Proc. Dynamic Flow Conference (Skovlunde, Denmark) pp. 757-800.

George, W. K., Beuther, P. D. \& Shabbir, A. 1989 Polynomial calibrations for hot wires in thermally varying flows. Experiment. Thermal Fluid Sci. 2, 230-235.

George, W. K. \& Gibson, M. M. 1992 The self preservation of homogeneous shear flow turbulence. Exps. in Fluids 13, 229-238.

Ghosal, S. \& Rogers, M. M. 1997 A numerical study of self-similarity in a turbulent plane wake using large-eddy simulation. Phys. Fluids 9, 1729-1739.

Gordeyev, S. \& Thomas, F. 2000 Coherent structures in the turbulent plane jet. Part 1. Extraction of proper orthogonal decomposition eigenmodes and their similarity. J. Fluid Mech. 414, 145-194.

Hinze, J. O. 1975 Turbulence. McGraw-Hill.

Hussein, H. J., Capp, S. P. \& George, W. K. 1994 Velocity measurements in a high-Reynoldsnumber, momentum-conserving, axisymmetric, turbulent jet. J. Fluid Mech. 258, 31-75.

IQBal, M. O. \& Thomas, F. O. 2007 Coherent structure in a turbulent jet via a vector implementation of the proper orthogonal decomposition. J. Fluid Mech. 571.

Johansson, P. B. V., George, W. K. \& Gourlay, M. J. 2003 Equilibrium similarity, effects of initial conditions and local Reynolds number on the axisymmetric wake. Phys. Fluids 15, 603-617.

Jung, D., Gamard, S. \& George, W. K. 2004 Downstream evolution of the most energetic modes in a turbulent axisymmetric jet at high Reynolds number. Part 1 . The near-field region. J. Fluid Mech. 514, 173-204.

VON KÁRmÉn, T. \& HowARTh, L. 1938 On the statistical theory of isotropic turbulence. Proc. R. Soc. Lond. A 164, 476.

Kopiev, V. F., Zaitsev, M. Y., Chernyshev, S. A. \& Kotova, A. N. 1999 The role of large-scale vortex in a turbulent jet noise. AIAA Paper 99-1839.

Mi, J., Nobis, D. S. \& Nathan, G. 2001 Influence of jet exit conditions on the passive scalar field of an axisymmetric free jet. J. Fluid Mech. 432, 91-125. 
Moser, R. D., Rogers, M. M. \& Ewing, D. 1998 Self-similarity of time-evolving plane wakes. J. Fluid Mech. 367, 255-289.

Mungal, M. G. \& Hollingsworth, D. K. 1989 Organized motion in a very high Reynolds number jet. Phys. Fluids 1, 1615-1623.

Panchapakesan, N. R. \& Lumley, J. L. 1993 Turbulence measurements in axisymmetric jets of air and helium. Part 1. Air jet. J. Fluid Mech. 246, 197-223.

Slessor, M. D., Bond, C. L. \& DimotaKis, P. E. 1998 Turbulent shear-layer mixing at high Reynolds numbers: Effects of initial conditions. J. Fluid Mech. 376, 115-138.

Speziale, C. G. \& Bernard, P. S. 1992 The energy decay in self-preserving isotropic turbulence revisited. J. Fluid Mech. 241, 645-667.

Tso, J. \& Hussain, F. 1989 Organized motions in a fully developed turbulent axisymmetric jet. J. Fluid Mech. 203, 425-448.

Wänström, M., George, W. K. \& Meyer, K. E. 2005 POD applied to stereo PIV data of the far turbulent axisymmetric jet. APS-DFD meeting, Chicago, IL, 2005, Bull. Amer. Phys. Soc. 50.

Westerweel, J., Hofmann, T., Fukushima, C. \& Hunt, J. C. R. 2002 The turbulent/non-turbulent interface at the outer boundary of a self-similar turbulent jet. Experiments in Fluids 33, 873-878.

Wygnanski, I., Champagne, F. \& Marasli, B. 1986 On the large-scale structures in two-dimensional small-deficit, turbulent wakes. J. Fluid Mech. 168, 31-71. 